\title{
Wave stability in anisotropic generalized temperature-rate-dependent thermoelasticity*
}

\author{
Amnah M. Alharbi \\ Department of Mathematics, Faculty of Science, Taif University, Taif, Saudi Arabia. \\ AND \\ NigEL H. SCOTT* \\ School of Mathematics, University of East Anglia, Norwich Research Park, Norwich NR4 \\ 7TJ, $U K$ \\ *Corresponding author: n.scott@uea.ac.uk
}

[Received on 9 May 2016]

\begin{abstract}
Temperature-rate-dependent thermoelasticity is a theory of thermoelasticity in which two relaxation times are introduced into the equations of classical thermoelasticity. An important consequence of this theory is that heat now travels at a finite speed rather than the infinite speed implied by the diffusion equation. In an anisotropic temperature-rate-dependent thermoelastic material it is found that four plane harmonic waves may propagate in any direction, all dispersive and attenuated, yet all are stable in the sense that their amplitudes remain bounded. An alternative theory that forces heat to travel at finite speed is generalized thermoelasticity in which the rate of change of heat flux also appears in the heat conduction equation, thereby introducing a relaxation time. Two different methods of combining the effects of temperaturerate-dependent thermoelasticity and generalized thermoelasticity are discussed and it is found that at least some of the four waves become unstable.
\end{abstract}

Keywords: Thermoelasticity, generalized thermoelasticity, second sound, harmonic waves, stability, two temperatures, two relaxation times.

\section{Introduction}

We are concerned in this paper with the stability of wave propagation in the Green \& Lindsay (1972) theory of temperature-rate-dependent thermoelasticity and in two of its extensions to include the generalized themoelasticity theory of Lord \& Shulman (1967). Both these theories evolved because the classical theory of thermoelasticity, see Chadwick (1979), effectively predicts that heating effects propagate with infinite speed which is regarded as being unrealistic from a physical point of view. This infinite speed arises because the differential equations of classical thermoelasticity are parabolic-hyperbolic in nature but it is replaced by a finite speed of propagation in both the Green \& Lindsay (1972) and Lord \& Shulman (1967) theories because both these theories give rise to purely hyperbolic systems of differential equations.

The classical Fourier law of heat conduction is

$$
q_{i}=-k_{i j} \theta_{, j}
$$

*Published as:

IMA Journal of Applied Mathematics (2016) 81, 750-778

doi:10.1093/imamat/hxw022 
in which $q_{i}(\mathbf{x}, t)$ are the components of the heat flux vector $\mathbf{q}, \theta(\mathbf{x}, t)$ is the temperature increment, both dependent on position $\mathbf{x}$ and time $t$, and $k_{i j}$ are the components of the heat conductivity tensor $\mathbf{k}$. The notation ()$,{ }_{j}$ denotes the spatial partial derivative $\partial() / \partial x_{j}$ and the summation convention applies so that twice-occurring suffixes are summed over.

The first theory of thermoelasticity to predict finite speeds for heating effects in thermoelasticity was that proposed by Lord \& Shulman (1967), who replaced Fourier's law (1.1) by the new law of heat conduction

$$
\left(1+\tau \frac{\partial}{\partial t}\right) q_{i}=-k_{i j} \theta_{, j}
$$

in which the constant $\tau>0$ is a relaxation time. Clearly, when $\tau=0$, equation (1.2) reduces to equation (1.1). The Lord \& Shulman (1967) theory is known as generalized thermoelasticity.

The second, proposed by Green \& Lindsay (1972), permits the stress to depend on the rate of change of temperature and the temperature gradient, thereby introducing two relaxation times. A noteworthy feature of this theory is that it preserves the classical Fourier law (1.1) if the material has a centre of symmetry at each point. The theory also demonstrates the symmetry of $\mathbf{k}$, that is, $k_{i j}=k_{j i}$. The Green \& Lindsay (1972) theory is known as temperature-rate-dependent thermoelasticity.

In his book on heat waves Straughan (2011) discusses both these theories together with other theories involving the propagation of heat effects at finite speed. The finite speed propagation of heat is also known as second sound. The development of second sound theories has been reviewed also by Chandrasekharaiah (1986).

Chandrasekharaiah \& Keshavan (1991) have introduced a certain amalgamation of the equations of temperature-rate-dependent thermoelasticity and generalized thermoelasticity in order to provide a unified system of governing equations for the two theories which we shall choose to explore as a possible model for generalized temperature-rate-dependent thermoelasticity. Theirs is an ad hoc system of equations which cannot be derived from any constitutive assumptions. Ignaczak (1980), on the other hand, provides a model for isotropic generalized temperature-rate-dependent thermoelasticity which is a rationally based combination of temperature-rate-dependent thermoelasticity and generalized thermoelasticity in the isotropic case. We generalize his results to the anisotropic case.

A small-amplitude wave form is said to be stable if it remains of uniformly bounded amplitude in the direction of propagation. In any material we might expect an initial small disturbance either to decay to zero or remain bounded as time increases. Thus we might regard the stability of wave forms as being a possible criterion for physically reasonable response. Scott (1989a) demonstrated the stability of anisotropic classical thermoelasticity and discussions of the isotropic case were given by Chadwick (1973) and Leslie \& Scott (1998). Scott (1989b) further demonstrated the stability of anisotropic generalized thermoelasticity with the isotropic case being discussed by Leslie \& Scott (2000).

The objective of the present paper is to utilise the techniques of the authors cited in the previous paragraph in order to investigate the stability of plane harmonic waves in each of the three theories introduced above, namely, the theories of Green \& Lindsay (1972), Chandrasekharaiah \& Keshavan (1991) and Ignaczak (1980). In Section 2 we derive the secular equation for plane harmonic waves in the temperature-rate-dependent thermoelasticity theory of Green \& Lindsay (1972). We find that there are four waves and that all are stable for all frequencies in the sense that they propagate with bounded amplitude. Similar calculations are performed in Section 3 for the Chandrasekharaiah \& Keshavan (1991) unified system of governing equations, considered here as a possible model for generalized temperature-rate-dependent thermoelasticity. We find that all four waves are stable at low frequencies but that at high frequencies at least one wave is unstable and another wave is stable with the other two being stable or unstable depending on the relative magnitudes of the various relaxation times. Simi- 
larly, in Section 4 for our generalization to anisotropy of the Ignaczak (1980) model of generalized temperature-rate-dependent thermoelasticity we find that all four waves are stable at low frequencies, as before, but that at high frequencies one wave is certainly unstable but the other three may be stable or unstable depending on the magnitude of the relaxation time associated with the heat flux. The final section contains a discussion of our results.

\section{Temperature-rate-dependent thermoelasticity}

We consider a thermoelastic body which possesses a spatially uniform, time-independent, stress-free equilibrium state free of heat flux. For a body with such an equilibrium state the equations of momentum and energy balance in the absence of body force and heat supply, linearized about this equilibrium state, are

$$
\sigma_{i j, j}=\rho \ddot{u}_{i}, \quad-q_{i, i}=\rho T \dot{\phi},
$$

respectively, see (Chadwick, 1979, Equations (16) and (17)), in which $\sigma_{i j}$ are the components of the Cauchy stress tensor and $\phi(\mathbf{x}, t)$ is the entropy increment per unit mass. The constant equilibrium values of the density and absolute temperature are denoted by $\rho$ and $T$, respectively, and the superposed dot denotes the time partial derivative.

The system of equations connecting the stress, entropy and heat flux are given implicitly by Green \& Lindsay (1972) and explicitly by (Straughan, 2011, Equations (2.57)) and (Chandrasekharaiah, 1986, Equations (5.11)-(5.13)) to be

$$
\begin{aligned}
& \sigma_{i j}=\tilde{c}_{i j k l} u_{k, l}-\beta_{i j}\left(1+\alpha_{1} \frac{\partial}{\partial t}\right) \theta \\
& \phi=\rho^{-1} \beta_{i j} u_{i, j}+T^{-1} c\left(1+\alpha_{0} \frac{\partial}{\partial t}\right) \theta-(\rho T)^{-1} d_{i} \theta_{, i}, \\
& q_{i}=-k_{i j} \theta_{, j}-d_{i} \dot{\theta}
\end{aligned}
$$

in which $\alpha_{0}, \alpha_{1}$ and $d_{i}$ are new material constants. The isothermal elasticity tensor components at constant equilibrium are denoted by $\tilde{c}_{i j k l}$, the temperature coefficients of stress are denoted by $\beta_{i j}$, and the specific heat at constant deformation is denoted by $c$. Green \& Lindsay (1972) show that the relaxation times $\alpha_{0}, \alpha_{1}$ satisfy the inequalities

$$
\alpha_{1} \geqslant \alpha_{0} \geqslant 0
$$

as a consequence of the second law of thermodynamics. They further observe that if the body has a centre of symmetry at each point then we may take $d_{i} \equiv 0$; we now make this assumption in common with most work on temperature-rate-dependent thermoelasticity. In order to deduce the field equations of temperature-rate-dependent thermoelasticity for an anisotropic material we need to insert (2.2) into (2.1) obtaining finally the field equations of temperature-rate-dependent thermoelasticity in the form

$$
\begin{aligned}
& \tilde{c}_{i j k l} u_{k, j l}-\beta_{i j}\left(\theta+\alpha_{1} \dot{\theta}\right)_{, j}=\rho \ddot{u}_{i}, \\
& k_{i j} \theta_{, i j}-T \beta_{i j} \dot{u}_{i, j}=\rho c\left(\dot{\theta}+\alpha_{0} \ddot{\theta}\right) .
\end{aligned}
$$

These equations form a complete system of four constant-coefficient, linear partial differential equations for the four unknown functions $u_{i}$ and $\theta$, see (Chandrasekharaiah, 1986, Equations (5.17)-(5.18)). By setting $\alpha_{1}=\alpha_{0}=0$, we recover the field equations

$$
\begin{aligned}
& \tilde{c}_{i j k l} u_{k, j l}-\beta_{i j} \theta_{, j}=\rho \ddot{u}_{i}, \\
& k_{i j} \theta_{, i j}-T \beta_{i j} \dot{u}_{i, j}=\rho c \dot{\theta},
\end{aligned}
$$


of classical thermoelasticity, see (Chadwick, 1979, Equation (19)).

In contrast with (2.4), the field equations of generalized thermoelasticity are given by

$$
\begin{aligned}
& \tilde{c}_{i j k l} u_{k, j l}-\beta_{i j} \theta_{, j}=\rho \ddot{u}_{i}, \\
& k_{i j} \theta_{, i j}-T \beta_{i j} \dot{u}_{i, j}=\rho c \dot{\theta}+\tau\left(\rho c \ddot{\theta}+T \beta_{i j} \ddot{u}_{i, j}\right),
\end{aligned}
$$

see (Scott, 1989b, Equation (2)). These equations may be obtained by taking $\alpha_{0}=\alpha_{1}=d_{i}=0$ in equations (2.2), replacing (2.2) 3 by the heat conduction equation (1.2) of generalized thermoelasticity and substituting the resulting equations into equations (2.1).

\subsection{The secular equation}

We are concerned with solutions of equations (2.4) in the form of plane waves

$$
\left\{u_{i}, \theta\right\}=\left\{U_{i}, \Theta\right\} \exp \{i \omega(s \mathbf{n} \cdot \mathbf{x}-t)\},
$$

where $\omega$ is the angular frequency and $\mathbf{n}$ is the unit wave normal vector in the direction of wave propagation, both of which are real constants. The amplitudes $\left\{U_{i}, \Theta\right\}$ and slowness $s$ are in general complex constants. The wave slowness $s$ is the reciprocal of the (complex) wave speed $v: s=1 / v$. We can derive the propagation conditions by inserting (2.7) into (2.4) and cancelling the exponential factor to give the linear system of algebraic equations

$$
\begin{aligned}
& \left(\tilde{c}_{i j k l} n_{j} n_{l}-\rho s^{-2} \delta_{i k}\right) U_{k}+i \omega^{-1} s^{-1} \beta_{i j} n_{j}\left(1-i \omega \alpha_{1}\right) \Theta=0, \\
& T s^{-1} \beta_{i j} n_{j} U_{i}+\left\{k_{i j} n_{i} n_{j}-i \omega^{-1}\left(1-i \omega \alpha_{0}\right) c \rho s^{-2}\right\} \Theta=0
\end{aligned}
$$

for the amplitudes $U_{i}$ and $\Theta$. We now introduce the isothermal and isentropic acoustic tensors, the scalar thermal conductivity and the vector $\mathbf{b}$, defined respectively by,

$$
\tilde{Q}_{i k}=\tilde{c}_{i j k l} n_{j} n_{l}, \quad \hat{Q}_{i k}=\hat{c}_{i j k l} n_{j} n_{l}, \quad k=k_{i j} n_{i} n_{j}, \quad b_{i}=\beta_{i j} n_{j} .
$$

The isentropic and isothermal elastic moduli are connected by

$$
\hat{c}_{i j k l}=\tilde{c}_{i j k l}+\frac{T}{\rho c} \beta_{i j} \beta_{k l},
$$

see (Chadwick, 1979, Equation (14)), leading to the connection

$$
\hat{Q}_{i k}=\tilde{Q}_{i k}+\frac{T}{\rho c} b_{i} b_{k}, \quad \text { or } \quad \hat{\mathbf{Q}}=\tilde{\mathbf{Q}}+\frac{T}{\rho c} \mathbf{b} \otimes \mathbf{b},
$$

see (Chadwick, 1979, Equation (24)), between the isentropic and isothermal acoustic tensors. We can rewrite (2.8), with aid of (2.9), as

$$
\begin{aligned}
& \left(\tilde{Q}_{i k}-\rho s^{-2} \delta_{i k}\right) U_{k}+i \omega^{-1} s^{-1} b_{i}\left(1-i \omega \alpha_{1}\right) \Theta=0 \\
& T s^{-1} b_{i} U_{i}+\left\{k-i \omega^{-1}\left(1-i \omega \alpha_{0}\right) c \rho s^{-2}\right\} \Theta=0 .
\end{aligned}
$$

In the Appendix we see how these equations may be manipulated to deduce the secular equation

$$
w \operatorname{det}\{\hat{\mathbf{Q}}-w \mathbf{1}\}+i \omega \frac{k / c+w\left(\alpha_{1}-\alpha_{0}\right)}{1-i \omega \alpha_{1}} \operatorname{det}\{\tilde{\mathbf{Q}}-w \mathbf{1}\}=0,
$$


in which $w=\rho s^{-2}$ is effectively a squared wave speed, see equation (A.2)1.

In order to non-dimensionalize equation (2.12), we introduce $\gamma$, a positive constant with the physical dimensions of stress (usually a typical component of the isothermal elasticities $\tilde{c}_{i j k l}$ ). In terms of $\gamma$, we define the frequency $\omega^{*}=\gamma c / k$. This leads to the following non-dimensional forms for the frequency $\omega$, relaxation times $\alpha_{0}$ and $\alpha_{1}$, squared wave speed $w$, and the isentropic and isothermal acoustic tensors $\hat{\mathbf{Q}}$ and $\tilde{\mathbf{Q}}$, respectively:

$$
\omega^{\prime}=\omega / \omega^{*}, \alpha_{0}^{\prime}=\alpha_{0} \omega^{*}, \alpha_{1}^{\prime}=\alpha_{1} \omega^{*}, w^{\prime}=w \gamma^{-1}, \hat{\mathbf{Q}}^{\prime}=\hat{\mathbf{Q}} \gamma^{-1}, \tilde{\mathbf{Q}}^{\prime}=\tilde{\mathbf{Q}} \gamma^{-1} .
$$

On dividing equation (2.12) by $\gamma^{4}$, and writing the resulting equation in terms of the non-dimensional quantities (2.13), we obtain the dimensionless secular equation

$$
w \operatorname{det}\{w \mathbf{1}-\hat{\mathbf{Q}}\}+i \omega \frac{1+w\left(\alpha_{1}-\alpha_{0}\right)}{1-i \omega \alpha_{1}} \operatorname{det}\{w \mathbf{1}-\tilde{\mathbf{Q}}\}=0,
$$

where we have dropped the dashes for convenience. Thus we have finally arrived at a non-dimensional form of the secular equation for anisotropic temperature-rate-dependent thermoelasticity.

On putting $\alpha_{1}=\alpha_{0}=0$ in equation (2.14) we recover the secular equation of anisotropic classical thermoelasticity, see (Chadwick, 1979, Equation (28)), as expected. It is of interest to note also that on putting $\alpha_{1}=\alpha_{0}$ in (2.13), and replacing both by $\tau$, there results the secular equation (Scott, 1989b, Equation (4)) of generalized thermoelasticity. In this sense, then, it can be said that generalized thermoelasticity is a special case of temperature-rate-dependent thermoelasticity. However, the relevant underlying field equations of temperature-rate-dependent thermoelasticity, namely (2.4), with $\alpha_{1}=\alpha_{0}$ and both replaced by $\tau$, are different from those of generalized thermoelasticity, see (2.6), even though the secular equations are the same.

Equation (2.14) is quartic in the squared wave speed $w$ with coefficients depending on the frequency $\omega$. The roots $w(\omega)$ of (2.14) represent the possible modes of wave propagation which form four branches in the complex $w$-plane as is illustrated later in the graphical results.

In terms of non-dimensional quantities the connection $(2.10)_{2}$ becomes

$$
\hat{\mathbf{Q}}=\tilde{\mathbf{Q}}+\varepsilon \overline{\mathbf{b}} \otimes \overline{\mathbf{b}},
$$

where the non-dimensional unit vector $\overline{\mathbf{b}}$ and the non-dimensional thermomechanical coupling constant $\varepsilon$ are defined, respectively, by

$$
\bar{b}_{i}=\frac{\beta_{i j} n_{j}}{\left\{\beta_{p q} n_{q} \beta_{p r} n_{r}\right\}^{1 / 2}}, \quad \varepsilon=\frac{T \beta_{p q} n_{q} \beta_{p r} n_{r}}{\rho c \gamma} .
$$

From the usual symmetries $\tilde{c}_{i j k l}=\tilde{c}_{k l i j}$ and $\hat{c}_{i j k l}=\hat{c}_{k l i j}$, see (Chadwick, 1979, Equations (9) and (14)), we see that both $\hat{\mathbf{Q}}$ and $\tilde{\mathbf{Q}}$ are symmetric and so each has three real eigenvalues. The nondimensional eigenvalues of $\hat{\mathbf{Q}}$ are denoted by $\hat{q}_{i}, i=1,2,3$, and the non-dimensional eigenvalues of $\tilde{\mathbf{Q}}$ are denoted by $\tilde{q}_{i}, i=1,2,3$. If these eigenvalues are distinct they possess the interlacing property

$$
0<\tilde{q}_{1}<\hat{q}_{1}<\tilde{q}_{2}<\hat{q}_{2}<\tilde{q}_{3}<\hat{q}_{3}
$$

as was demonstrated by Scott (1989a). The inequality $0<\tilde{q}_{1}$ follows from the assumed positive definiteness of $\tilde{\mathbf{Q}}$, see Scott (1989a). If some or all of these eigenvalues coincide then some or all of the other occurrences of $<$ in (2.17) may be replaced by $\leqslant$, see Scott (1989a) for a discussion of coincident eigenvalues. 
Taking the trace of (2.15) gives the following connection between the coupling constant and the eigenvalues of $\hat{\mathbf{Q}}$ and $\tilde{\mathbf{Q}}$ :

$$
\varepsilon=\hat{q}_{1}+\hat{q}_{2}+\hat{q}_{3}-\tilde{q}_{1}-\tilde{q}_{2}-\tilde{q}_{3} .
$$

From inequalities (2.17) we see that

$$
\varepsilon>0
$$

which may be replaced by the equality

$$
\varepsilon=0
$$

only if the isothermal and isentropic eigenvalues coincide in pairs, i.e. $\tilde{q}_{i}=\hat{q}_{i}$ for $i=1,2,3$.

We may rewrite the secular equation (2.14) in terms of the eigenvalues $\hat{q}_{i}$ and $\tilde{q}_{i}, i=1,2,3$, as follows

$$
\hat{F}(w)+i \omega \frac{1+w\left(\alpha_{1}-\alpha_{0}\right)}{1-i \omega \alpha_{1}} \tilde{G}(w)=0
$$

where we define

$$
\hat{F}(w)=w \prod_{i=1}^{3}\left(w-\hat{q}_{i}\right), \quad \tilde{G}(w)=\prod_{i=1}^{3}\left(w-\tilde{q}_{i}\right)
$$

2.1.1 Stability considerations We seek a simple condition for a plane wave mode of the form (2.7) to be stable in the sense that its amplitude should remain bounded in the direction of travel. We expand the exponential in the plane wave mode (2.7) as

$$
\exp \{i \omega(s \mathbf{n} \cdot \mathbf{x}-t)\}=\exp \left\{i \omega\left(s_{+} \mathbf{n} \cdot \mathbf{x}-t\right)\right\} \exp \left(-\omega s_{-} \mathbf{n} \cdot \mathbf{x}\right)
$$

where $s_{+}$and $s_{-}$are the real and imaginary parts of the complex slowness

$$
s=s_{+}+i s_{-} .
$$

The first exponential on the right-hand side of (2.23) corresponds to a constant-amplitude sinusoidal wave travelling with speed $1 / s_{+}$and the second to either exponential decay or increase. We require the wave form to be bounded in $\mathbf{x}$ as $s_{+} \mathbf{n} \cdot \mathbf{x} \rightarrow+\infty$ when $t \rightarrow+\infty$, so that either $\mathbf{n} \cdot \mathbf{x} \rightarrow+\infty$ for $s_{+}>0$ or $\mathbf{n} \cdot \mathbf{x} \rightarrow-\infty$ for $s_{+}<0$. Exponential decay in the first case necessitates $s_{-}>0$ and in the second $s_{-}<0$, provided the frequency $\omega$ is real and positive. The case $s_{+}=0$ is excluded as it does not correspond to a travelling wave. Therefore the stability of the wave form (2.7) requires

$$
s_{+} s_{-} \geqslant 0
$$

in which $s_{-}=0$ is permissible but $s_{+}=0$ is not. Written in terms of the dimensionless squared wave speed $w=\rho s^{-2} / \gamma$ the above stability condition becomes

$$
\operatorname{Im} w(\omega) \leqslant 0,
$$

for $0 \leqslant \omega<\infty$, see also (Scott, 1989a, Equation (18)). The positive real axis of the complex $w$-plane is included in the region of stability but the negative real axis is not as it corresponds to $s_{+}=0$. So for positive $\omega$, stable branches $w(\omega)$ are those which lie in the lower half of the complex $w$-plane. On the other hand, it can be shown that for negative frequency, stability requires the inequality in (2.24) to be reversed, so that for negative $\omega$ the stable branches of $w(\omega)$ are those which lie in the upper half of the complex $w$-plane.

Each branch of the secular equation (2.21) is examined in detail below, with low- and high-frequency expansions being performed, and stability proved for the entire frequency range. 
2.1.2 The uncoupled case $\varepsilon=0$. In this case $\tilde{q}_{i}=\hat{q}_{i}$ for $i=1,2,3$, see just after equation (2.20), so that the functions $\hat{F}(w)$ and $\tilde{G}(w)$ of (2.22) have three factors in common. Then the roots of (2.21) reduce to

$$
w_{0}=\frac{-i \omega}{1-i \omega \alpha_{0}}, \quad w_{i}=\tilde{q}_{i}=\hat{q}_{i}, \quad i=1,2,3
$$

where $w_{0}$ represents a purely diffusive mode and $w_{i}, i=1,2,3$, represent unattenuated, non-dispersive waves (purely elastic modes). We can show that as $\omega$ varies over positive values the branch $w_{0}$ describes a semicircle in the lower half $w$-plane if $\alpha_{0}>0$ with centre at $\alpha_{0} / 2+0 i$ and radius $\alpha_{0} / 2$.

\subsection{Low-frequency expansions}

When $\omega=0$, the roots of the secular equation (2.21) are the zeros of $\hat{F}(w)$, namely, $w=\hat{q}_{i}, i=$ $0,1,2,3$, defining $\hat{q}_{0} \equiv 0$. Taylor expansions about the roots $\hat{q}_{i}$ of the secular equation (2.21) for small $\omega$ take the form

$$
w_{i}(\omega)=\hat{q}_{i}-i \omega\left\{1+\hat{q}_{i}\left(\alpha_{1}-\alpha_{0}\right)\right\} \frac{\tilde{G}\left(\hat{q}_{i}\right)}{\hat{F}^{\prime}\left(\hat{q}_{i}\right)}+O\left(\omega^{2}\right), \quad i=0,1,2,3 .
$$

When $i=0$, so that $\hat{q}_{0}=0$, the appropriate branch of $(2.26)$ is

$$
w_{0}(\omega)=-i \omega \frac{\tilde{G}(0)}{\hat{F}^{\prime}(0)}=-i \omega \frac{\tilde{q}_{1} \tilde{q}_{2} \tilde{q}_{3}}{\hat{q}_{1} \hat{q}_{2} \hat{q}_{3}}
$$

ignoring $O\left(\omega^{2}\right)$ terms, which is clearly stable since $\operatorname{Im} w_{0}<0$. In fact, the interlacing conditions (2.17) suffice to show that

$$
\frac{\tilde{G}\left(\hat{q}_{i}\right)}{\hat{F}^{\prime}\left(\hat{q}_{i}\right)}>0, \quad i=0,1,2,3
$$

It is clear from (2.3), (2.26) and (2.28) that the condition of stability (2.24) is confirmed for each branch. Therefore, there are four stable waves in the low-frequency limit.

\subsection{High-frequency expansions}

When $\omega \rightarrow \infty$, the roots of the secular equation (2.21) are given by the zeros of $H(w)$ where

$$
H(w) \equiv \hat{F}(w)-\frac{1}{\alpha_{1}}\left\{1+w\left(\alpha_{1}-\alpha_{0}\right)\right\} \tilde{G}(w)
$$

in which $\hat{F}(w)$ and $\tilde{G}(w)$ are defined at (2.22). It is clear that $H(w)$ is quartic in $w$ provided that $\alpha_{0} \neq 0$, so there are four zeros, denoted by $\bar{q}_{1}, \bar{q}_{2}, \bar{q}_{3}, \bar{q}_{4}$. In order to locate these zeros we need to examine the 
sign changes of $H(w)$. By using the inequalities (2.17) and the definition (2.29) we find that

$$
\begin{aligned}
& H(0)=-\alpha_{1}^{-1}\left(-\tilde{q}_{1}\right)\left(-\tilde{q}_{2}\right)\left(-\tilde{q}_{3}\right)>0, \\
& H\left(\tilde{q}_{1}\right)=\tilde{q}_{1}\left(\tilde{q}_{1}-\hat{q}_{1}\right)\left(\tilde{q}_{1}-\hat{q}_{2}\right)\left(\tilde{q}_{1}-\hat{q}_{3}\right)<0, \\
& H\left(\hat{q}_{1}\right)=-\alpha_{1}^{-1}\left\{1+\hat{q}_{1}\left(\alpha_{1}-\alpha_{0}\right)\right\}\left(\hat{q}_{1}-\tilde{q}_{1}\right)\left(\hat{q}_{1}-\tilde{q}_{2}\right)\left(\hat{q}_{1}-\tilde{q}_{3}\right)<0, \\
& H\left(\tilde{q}_{2}\right)=\tilde{q}_{2}\left(\tilde{q}_{2}-\hat{q}_{1}\right)\left(\tilde{q}_{2}-\hat{q}_{2}\right)\left(\tilde{q}_{2}-\hat{q}_{3}\right)>0, \\
& H\left(\hat{q}_{2}\right)=-\alpha_{1}^{-1}\left\{1+\hat{q}_{2}\left(\alpha_{1}-\alpha_{0}\right)\right\}\left(\hat{q}_{2}-\tilde{q}_{1}\right)\left(\hat{q}_{2}-\tilde{q}_{2}\right)\left(\hat{q}_{2}-\tilde{q}_{3}\right)>0, \\
& H\left(\tilde{q}_{3}\right)=\tilde{q}_{3}\left(\tilde{q}_{3}-\hat{q}_{1}\right)\left(\tilde{q}_{3}-\hat{q}_{2}\right)\left(\tilde{q}_{3}-\hat{q}_{3}\right)<0, \\
& H\left(\hat{q}_{3}\right)=-\alpha_{1}^{-1}\left\{1+\hat{q}_{3}\left(\alpha_{1}-\alpha_{0}\right)\right\}\left(\hat{q}_{3}-\tilde{q}_{1}\right)\left(\hat{q}_{3}-\tilde{q}_{2}\right)\left(\hat{q}_{3}-\tilde{q}_{3}\right)<0, \\
& H(\infty)=\infty>0 .
\end{aligned}
$$

From the changes of sign of $H(w)$ recorded in inequalities (2.30) we see that the $\bar{q}_{i}$ interlace according to

$$
0<\bar{q}_{1}<\tilde{q}_{1}<\hat{q}_{1}<\bar{q}_{2}<\tilde{q}_{2}<\hat{q}_{2}<\bar{q}_{3}<\tilde{q}_{3}<\hat{q}_{3}<\bar{q}_{4} .
$$

Eliminating $\hat{F}(w)$ between (2.21) and (2.29) gives the secular equation in the form

$$
H(w)+\frac{1+w\left(\alpha_{1}-\alpha_{0}\right)}{\alpha_{1}\left(1-i \omega \alpha_{1}\right)} \tilde{G}(w)=0 .
$$

The quartic polynomial

$$
\bar{h}(w)=\left(w-\bar{q}_{1}\right)\left(w-\bar{q}_{2}\right)\left(w-\bar{q}_{3}\right)\left(w-\bar{q}_{4}\right),
$$

must be a scalar multiple of $H(w)$ because both have the same four zeros. By comparing coefficients of $w^{4}$ we see that

$$
H(w) \equiv \frac{\alpha_{0}}{\alpha_{1}} \bar{h}(w)
$$

Inserting (2.33) into (2.32) we obtain the secular equation (2.21) in the form

$$
\alpha_{0}\left(1-i \omega \alpha_{1}\right) \bar{h}(w)+\left\{1+w\left(\alpha_{1}-\alpha_{0}\right)\right\} \tilde{G}(w)=0,
$$

valid for all $\omega$.

Taylor expansions about the roots $\bar{q}_{i}$ of the secular equation (2.34) in the high-frequency limit, i.e. for $\omega^{-1}$ small, reveal that

$$
w_{i}(\omega)=\bar{q}_{i}-\frac{i \omega^{-1}}{\alpha_{0} \alpha_{1}}\left\{1+\bar{q}_{i}\left(\alpha_{1}-\alpha_{0}\right)\right\} \frac{\tilde{G}\left(\bar{q}_{i}\right)}{\bar{h}^{\prime}\left(\bar{q}_{i}\right)}+O\left(\omega^{-2}\right), \quad i=1,2,3,4,
$$

with the interlacing properties (2.31) guaranteeing that

$$
\frac{\tilde{G}\left(\bar{q}_{i}\right)}{\bar{h}^{\prime}\left(\bar{q}_{i}\right)}>0, \quad i=1,2,3,4 .
$$

From inequality (2.36) and the fact that $\alpha_{1} \geqslant \alpha_{0}$ we see that the stability condition (2.24) is satisfied, so that there are four stable waves in the high-frequency limit. 


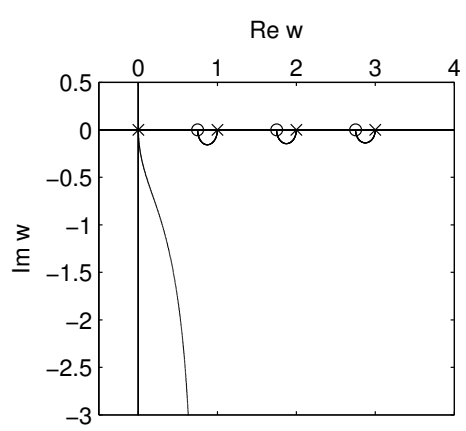

(a) $\alpha_{0}=0, \alpha_{1}=0$

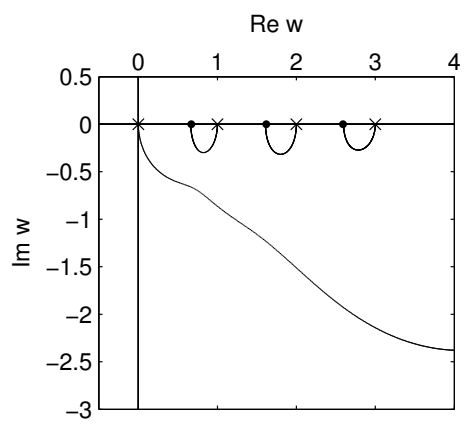

(c) $\alpha_{0}=0.2, \alpha_{1}=0.3$

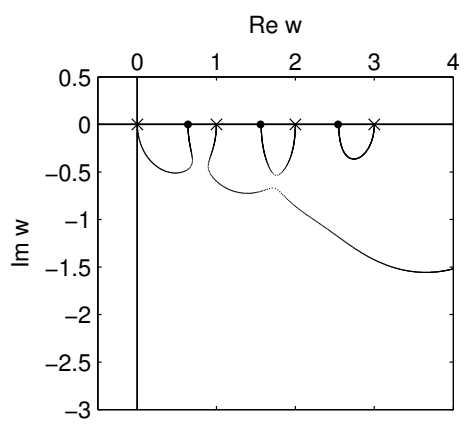

(e) $\alpha_{0}=0.27, \alpha_{1}=0.37$

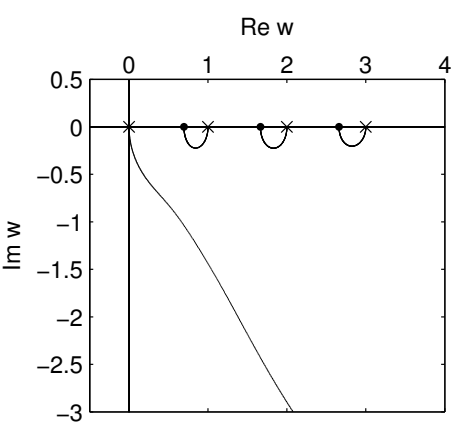

(b) $\alpha_{0}=0.1, \alpha_{1}=0.2$

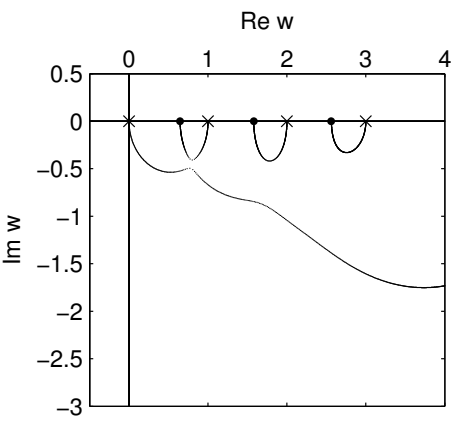

(d) $\alpha_{0}=0.25, \alpha_{1}=0.35$

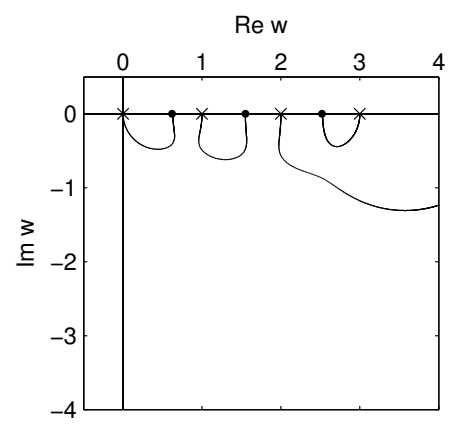

(f) $\alpha_{0}=0.3, \alpha_{1}=0.4$

FIG. 1. The four branches of the secular equation for anisotropic temperature-rate-dependent thermoelasticity. The case $\alpha_{1}>\alpha_{0}$. For each part, $\tilde{q}_{1}=0.75, \tilde{q}_{2}=1.75, \tilde{q}_{3}=2.75, \hat{q}_{1}=1, \hat{q}_{2}=2, \hat{q}_{3}=3$. 
10 of 26

A. M. ALHARBI AND N. H. SCOTT

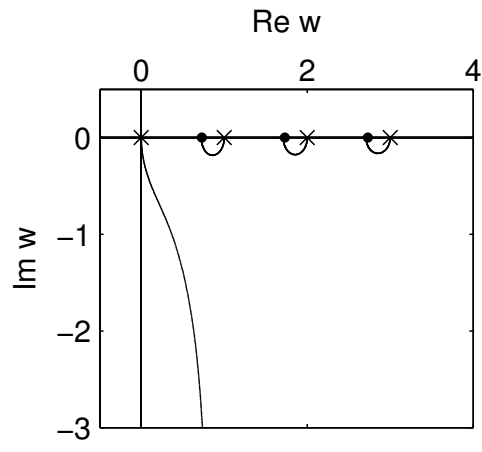

(a) $\alpha_{0}=0, \alpha_{1}=0.1$

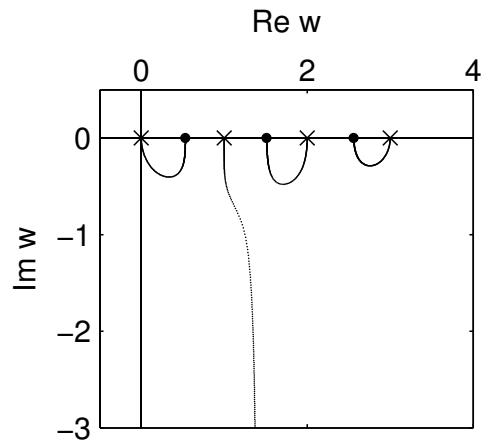

(c) $\alpha_{0}=0, \alpha_{1}=1$

Re w

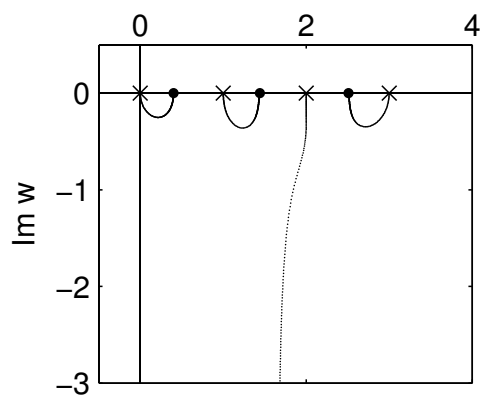

(e) $\alpha_{0}=0, \alpha_{1}=2$

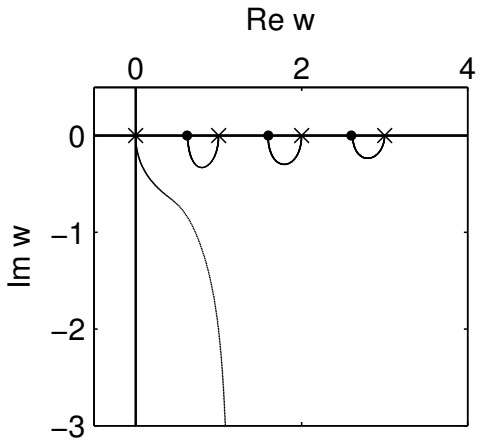

(b) $\alpha_{0}=0, \alpha_{1}=0.5$

Re w

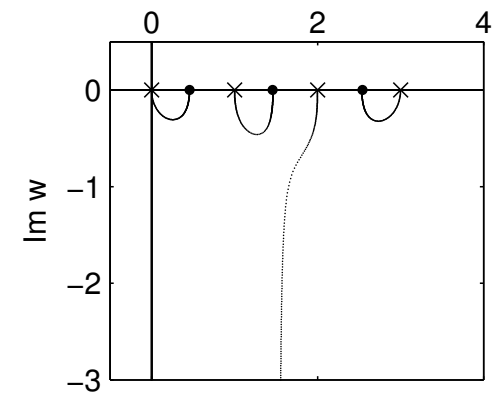

(d) $\alpha_{0}=0, \alpha_{1}=1.5$

Re w

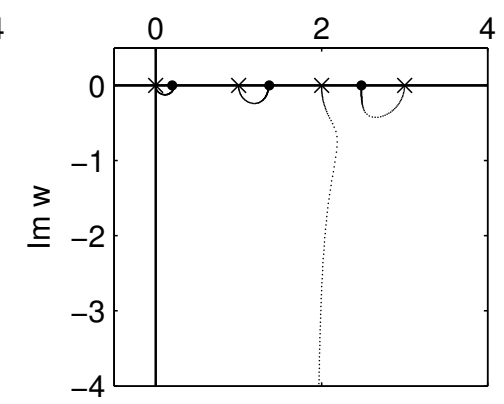

(f) $\alpha_{0}=0, \alpha_{1}=5$

FIG. 2. The four branches of the secular equation for anisotropic temperature-rate-dependent thermoelasticity. The case $\alpha_{0}=$ $0, \alpha_{1}>0$. For each part, $\tilde{q}_{1}=0.75, \tilde{q}_{2}=1.75, \tilde{q}_{3}=2.75, \hat{q}_{1}=1, \hat{q}_{2}=2, \hat{q}_{3}=3$. 


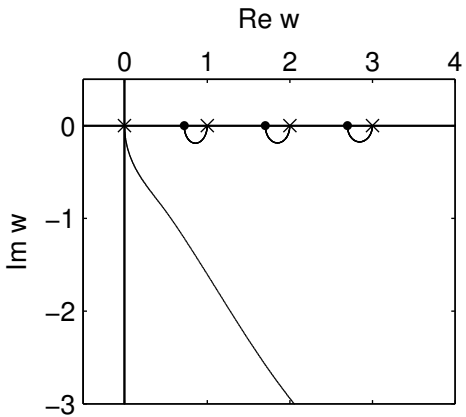

(a) $\alpha_{0}=\alpha_{1}=0.1$

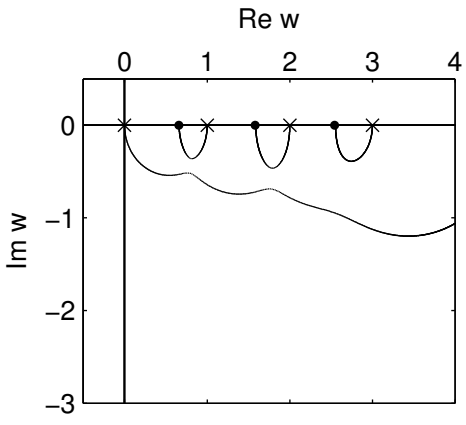

(c) $\alpha_{0}=\alpha_{1}=0.29$

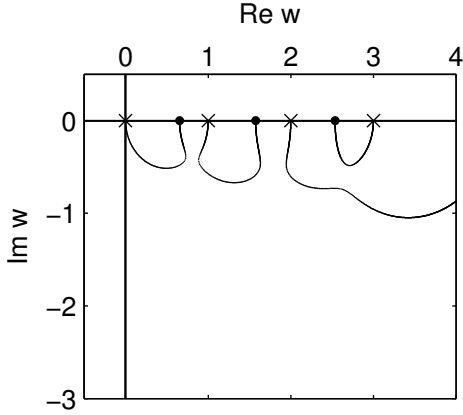

(e) $\alpha_{0}=\alpha_{1}=0.31$

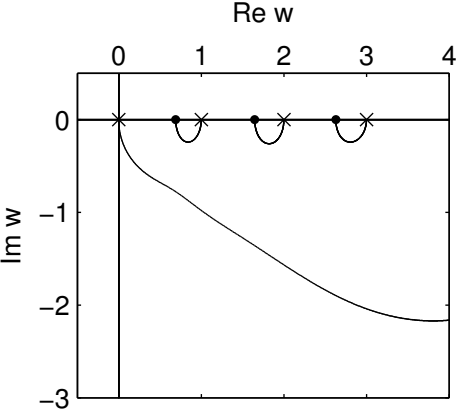

(b) $\alpha_{0}=\alpha_{1}=0.2$

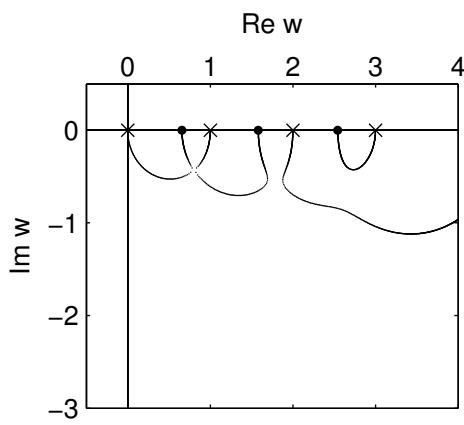

(d) $\alpha_{0}=\alpha_{1}=0.3$

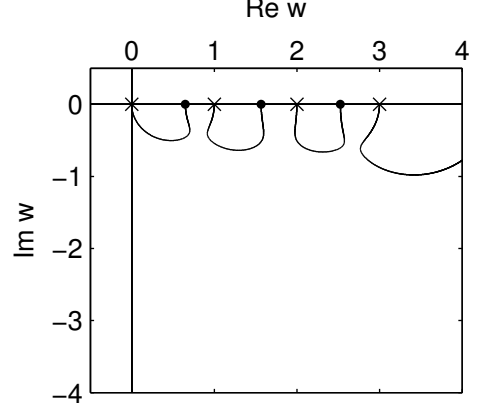

(f) $\alpha_{0}=\alpha_{1}=0.32$

FIG. 3. The four branches of the secular equation for anisotropic temperature-rate-dependent thermoelasticity. The case $\alpha_{1}=$ $\alpha_{0}>0$. For each part, $\tilde{q}_{1}=0.75, \tilde{q}_{2}=1.75, \tilde{q}_{3}=2.75, \hat{q}_{1}=1, \hat{q}_{2}=2, \hat{q}_{3}=3$.

\subsection{Stability for all frequencies}

We have shown that all four branches are stable in the low- and high-frequency limits. In order to prove the stability of each one throughout the entire frequency range $0<\omega<\infty$, we must prove that a branch may cut the real axis only at the low- and high-frequency limits. If this were not true we would be able 
to solve the secular equation (2.21) for real $w$ and some $\omega$ in the range $0<\omega<\infty$. Rearrange (2.21) into the form

$$
\frac{\hat{F}(w)}{\left\{1+w\left(\alpha_{1}-\alpha_{0}\right)\right\} \tilde{G}(w)}=\frac{-i \omega}{1-i \omega \alpha_{1}} .
$$

For real $w$ the left-hand side of (2.37) is real and so cannot be equal to the (necessarily) complex righthand side for any $\omega$ in the range $0<\omega<\infty$. Thus, all branches are stable for all frequencies $0 \leqslant \omega<\infty$.

\subsection{Numerical results}

The low-frequency branch starting from the origin and any part of a high-frequency branch tending to infinity in the complex $w$-plane are named diffusive modes. On the other hand, any other branches that begin and end close to the real axis, away from the origin, are named elastic modes, see Chadwick (1973). A branch of $w(\omega)$ such that $w(\omega) \rightarrow \infty$ as $\omega \rightarrow \infty$ is termed an infinite branch.

Figure 1 shows an example for different values of $\alpha_{1}$ and $\alpha_{0}$ such that $\alpha_{1} \geqslant \alpha_{0} \geqslant 0$, demonstrating the effect of $\alpha_{1}$ and $\alpha_{0}$ increasing. In each sub-figure we select the same values of $\hat{q}_{i}$ and $\tilde{q}_{i}, i=1,2,3$. The low-frequency limits are marked with $a \times$ and the high-frequency limits with $a \circ$ in the first subfigure, which corresponds to classical thermoelasticity, and with $\bullet$ in the others which correspond to temperature-rate-dependent-thermoelasticity. It is clear that all branches lie in the lower half of the complex $w$-plane and so satisfy the stability condition (2.24).

Fig. 1, part (a) represents classical thermoelasticity and all branches of $w(\omega)$ are stable but three of them are finite and one is infinite. However, the other parts (b)-(f) for temperature-rate-dependentthermoelasticity show that the existence of relaxation times $\alpha_{0}$ and $\alpha_{1}$ maintains stability and makes all branches finite.

In Figure 2, in which $\alpha_{0}=0$ and $\alpha_{1}>0$, we see that the four branches are always stable, but three of them are finite and one is infinite. This is because for $\alpha_{0}=0, H(w)$ defined by (2.29) becomes cubic in $w$, rather than quartic, with the result that the fourth root $\bar{h}_{4}$ changes character from a finite positive real value to a large negative imaginary value. For $\alpha_{0}$ small we can show that the fourth high-frequency root of (2.29) is large and positive, in fact $\bar{q}_{4} \approx\left(1+\varepsilon \alpha_{1}\right) / \alpha_{0}$, so that in Figure $1(\mathrm{~b})$, for example, $\bar{q}_{4} \approx 11.5$.

The equations of this section greatly simplify in the special case $\alpha_{1}=\alpha_{0}>0$ but the analysis remains valid, as is illustrated in Figure 3. In sub-figure (d) two branches appear to intersect.

\section{Generalized temperature-rate-dependent thermoelasticity: Model 1}

Chandrasekharaiah \& Keshavan (1991) combined the field equations of temperature-rate-dependent thermoelasticity (2.4) with the modified heat conduction equation (1.2) of generalized thermoelasticity in order to arrive at a unified system of equations for studying the two theories together, see equations (3.1) below. We propose to study this unified system as a possible model for generalized temperaturerate-dependent thermoelasticity which we shall refer to as generalized temperature-rate-dependent thermoelasticity: Model 1, although Chandrasekharaiah \& Keshavan (1991) did not regard their unified system of equations as such.

The system of equations for generalized temperature-rate-dependent thermoelasticity: Model 1 proposed by (Chandrasekharaiah \& Keshavan, 1991, Equation (2.3)) is

$$
\begin{aligned}
& \tilde{c}_{i j k l} u_{k, j l}-\beta_{i j}\left(1+\alpha_{1} \frac{\partial}{\partial t}\right) \theta_{, j}=\rho \ddot{u}_{i}, \\
& k_{i j} \theta_{, i j}-\left(1+\tau_{0} \frac{\partial}{\partial t}\right) T \beta_{i j} \dot{u}_{i, j}=\rho c\left(1+\alpha_{0} \frac{\partial}{\partial t}\right) \dot{\theta} .
\end{aligned}
$$


The first of these equations is exactly the same as equation (2.4) $)_{1}$ of temperature-rate-dependent thermoelasticity but the second is a modified form of $(2.4)_{2}$. The new relaxation time $\tau_{0}$ has been introduced so that putting $\tau_{0}=0$ in equations (3.1) reduces them to equations (2.4) of temperature-rate-dependent thermoelasticity. Putting $\alpha_{1}=0$, with $\alpha_{0}=\tau_{0}>0$, in (3.1) reduces these equations to those of generalized thermoelasticity, see (Scott, 1989b, Equation (4)).

The above choice of relaxation times, however, involves taking $\alpha_{1}<\alpha_{0}$ which contravenes inequality (2.3) of temperature-rate-dependent thermoelasticity, thus exposing the ad hoc nature of the system (3.1). There is no choice of constitutive assumptions for stress, entropy and heat flux which gives rise to the system (3.1).

\subsection{The secular equation}

We use the form of plane harmonic waves (2.7) to reduce (3.1) to the linear algebraic system

$$
\begin{aligned}
& \left(\tilde{Q}_{i k}-\rho s^{-2} \delta_{i k}\right) U_{k}+i \omega^{-1} s^{-1} b_{i}\left(1-i \omega \alpha_{1}\right) \Theta=0, \\
& T s^{-1}\left(1-i \omega \tau_{0}\right) b_{i} U_{i}+\left\{k-i \omega^{-1}\left(1-i \omega \alpha_{0}\right) c \rho s^{-2}\right\} \Theta=0 .
\end{aligned}
$$

These are exactly the same as (2.11) in the previous section except for the occurrence of the factor $\left(1-i \omega \tau_{0}\right)$ in the second equation.

Using methods similar to the previous section and using the dimensionless quantities (2.13), with the further non-dimensional relaxation time

$$
\tau=\tau_{0} \omega^{*}
$$

we find that the secular equation may be written in non-dimensional form as

$$
\hat{F}(w)+i \omega \frac{1+w\left\{\alpha_{1}-\alpha_{0}+\tau\left(1-i \omega \alpha_{1}\right)\right\}}{\left(1-i \omega \alpha_{1}\right)(1-i \omega \tau)} \tilde{G}(w)=0,
$$

with $\hat{F}(w)$ and $\tilde{G}(w)$ defined earlier at (2.22). This is the dimensionless secular equation of generalized temperature-rate-dependent thermoelasticity: Model 1.

For $\tau=0$, (3.4) reduces to (2.21), as expected. In the generalized thermoelasticity limit, where $\alpha_{1}=0$ and $\alpha_{0}=\tau>0$, the secular equation (3.4) reduces to the anisotropic generalized thermoelasticity secular equation, see (Scott, 1989b, Equation (4)).

3.1.1 The uncoupled case $\varepsilon=0$. In this case, the roots of the secular equation (3.4) reduce to those of the purely temperature-rate-dependent case, namely, equations (2.25).

\subsection{Low-frequency expansions}

When $\omega=0$, the roots of the secular equation (3.4) are the zeros of $\hat{F}(w)$, namely, $w=\hat{q}_{i}, i=0,1,2,3$, defining $\hat{q}_{0} \equiv 0$, exactly as in the previous section. Taylor expansions about each of the roots $\hat{q}_{i}$ of the secular equation (3.4) for small $\omega$ give

$$
w_{i}(\omega)=\hat{q}_{1}-i \omega\left\{1+\hat{q}_{1}\left(\alpha_{1}-\alpha_{0}+\tau\right)\right\} \frac{\tilde{G}\left(\hat{q}_{i}\right)}{\hat{F}^{\prime}\left(\hat{q}_{i}\right)}+O\left(\omega^{2}\right), \quad i=0,1,2,3 .
$$


When $i=0$, so that $\hat{q}_{0}=0$, the appropriate branch of (3.5) is

$$
w_{0}(\omega)=-i \omega \frac{\tilde{G}(0)}{\hat{F}^{\prime}(0)}=-i \omega \frac{\tilde{q}_{1} \tilde{q}_{2} \tilde{q}_{3}}{\hat{q}_{1} \hat{q}_{2} \hat{q}_{3}},
$$

ignoring $O\left(\omega^{2}\right)$ terms, which is clearly stable since $\operatorname{Im} w_{0}<0$. The branch $w_{0}(\omega)$ given by (3.6) is exactly the same as that of the purely temperature-rate-dependent case, see (2.27).

It is clear from the inequalities (2.3) and (2.28) that $\operatorname{Im} w_{i}(\omega)<0$, which means the condition of stability (2.24) is satisfied for all four modes. So there are four stable waves in the low-frequency limit.

\subsection{High-frequency expansions}

The roots of the secular equation (3.4) in the high-frequency limit $\omega \rightarrow \infty$ are best examined by writing $\omega=1 / \zeta$ and taking the limit $\zeta \rightarrow 0$. Equation (3.4) becomes in terms of $\zeta$

$$
\hat{F}(w)-\frac{w \alpha_{1} \tau+i \zeta\left\{1+w\left(\alpha_{1}-\alpha_{0}+\tau\right)\right\}}{\alpha_{1} \tau\left(1+i \zeta / \alpha_{1}\right)(1+i \zeta / \tau)} \tilde{G}(w)=0,
$$

valid for all $\zeta$. At $\zeta=0,(3.7)$ reduces to

$$
H(w) \equiv \hat{F}(w)-w \tilde{G}(w)=0,
$$

the solutions of which are the infinite frequency roots of the secular equation (3.7). This equation is not quartic in $w$ as the $w^{4}$ terms cancel out. It is a cubic equation in $w$ with one root $w=0$ denoted by $\bar{q}_{1}=0$ and the other roots denoted by $\bar{q}_{2}$ and $\bar{q}_{3}$ with $\bar{q}_{2}<\bar{q}_{3}$. By examining the sign changes of $H(w)$ as we did at (2.30), bearing in mind the interlacings (2.17), we discover the further interlacing properties

$$
0=\bar{q}_{1}<\tilde{q}_{1}<\hat{q}_{1}<\bar{q}_{2}<\tilde{q}_{2}<\hat{q}_{2}<\bar{q}_{3}<\tilde{q}_{3}<\hat{q}_{3} .
$$

The cubic polynomial

$$
\bar{h}(w)=w\left(w-\bar{q}_{2}\right)\left(w-\bar{q}_{3}\right)
$$

must be a scalar multiple of $H(w)$ because both have same three zeros:

$$
H(w)=-\varepsilon \bar{h}(w)
$$

since the coefficient of $w^{3}$ in $H(w)$ is $-\varepsilon$, where $\varepsilon$ is given by (2.18).

By using (3.8), (3.10) and (3.11) we find that the secular equation (3.7) becomes

$$
\bar{h}(w)+\frac{i \zeta}{\varepsilon \alpha_{1} \tau} \frac{1-\alpha_{0} w-i \zeta w}{\left(1+i \zeta / \alpha_{1}\right)(1+i \zeta / \tau)} \tilde{G}(w)=0,
$$

valid for all $\zeta$. To find approximations to the roots for small $\zeta$ we need only go as far as terms in $O(i \zeta)$ in (3.12) and so this equation may be simplified to

$$
\bar{h}(w)+\frac{i \zeta}{\varepsilon \alpha_{1} \tau}\left(1-\alpha_{0} w\right) \tilde{G}(w)+O\left(\zeta^{2}\right)=0 .
$$

At $\zeta=0,(3.13)$ has the three roots $\bar{q}_{i}, i=1,2,3$, which are the zeros of (3.10), so for small $\zeta$ there are three branches $w_{i}, i=1,2,3$. Reverting to the frequency $\omega=\zeta^{-1}$, power series expansions of these branches reveal that

$$
w_{i}(\omega)=\bar{q}_{i}-i \omega^{-1} \frac{1-\alpha_{0} \bar{q}_{i}}{\varepsilon \alpha_{1} \tau} \frac{\tilde{G}\left(\bar{q}_{i}\right)}{\bar{h}^{\prime}\left(\bar{q}_{i}\right)}+O\left(\omega^{-2}\right), \quad i=1,2,3
$$


and from the interlacing inequalities (3.9) we find that

$$
\frac{\tilde{G}\left(\bar{q}_{i}\right)}{\bar{h}^{\prime}\left(\bar{q}_{i}\right)}<0, \quad i=1,2,3 .
$$

For the branch $i=1$, for which $\bar{q}_{1}=0,(3.14)$ reduces to

$$
w_{1}(\omega)=-\frac{i \omega^{-1}}{\varepsilon \alpha_{1} \tau} \frac{\tilde{G}(0)}{\bar{h}^{\prime}(0)}=\frac{i \omega^{-1}}{\varepsilon \alpha_{1} \tau} \frac{\tilde{q}_{1} \tilde{q}_{2} \tilde{q}_{3}}{\bar{q}_{1} \bar{q}_{2}}
$$

ignoring $O\left(\omega^{-2}\right)$ terms. Clearly, $\operatorname{Im} w_{1}(\omega)>0$ and so $w_{1}$ is unstable.

From the ordering $\bar{q}_{2}<\bar{q}_{3}$ and (3.14) and (3.15) we see that the stability or otherwise of the branches $w_{2}$ and $w_{3}$ is governed entirely by the magnitude of $\alpha_{0}$ according to

$$
\begin{array}{ll}
\alpha_{0}>1 / \bar{q}_{2}>1 / \bar{q}_{3}, & w_{2} \text { and } w_{3} \text { are both stable, } \\
1 / \bar{q}_{2}>\alpha_{0}>1 / \bar{q}_{3}, & w_{2} \text { is unstable and } w_{3} \text { is stable, } \\
1 / \bar{q}_{2}>1 / \bar{q}_{3}>\alpha_{0} \geqslant 0, & w_{2} \text { and } w_{3} \text { are both unstable. }
\end{array}
$$

Since (3.13) is quartic in $w$ when $\zeta>0$, it must have a fourth branch, $w_{4}$, which is large when $\zeta$ is small. This can be written as

$$
w_{4}=A(i \zeta)^{-1}+B+O(i \zeta)
$$

where $A$ and $B$ are constants. We substitute (3.18) into (3.13), multiply by $(i \zeta)^{3}$ in order to remove all negative powers of $i \zeta$, and obtain from the constant terms of the ensuing equation, the quartic equation

$$
A^{3}-\frac{\alpha_{0}}{\varepsilon \alpha_{1} \tau} A^{4}=0
$$

with roots

$$
A=0,0,0, \frac{\varepsilon \alpha_{1} \tau}{\alpha_{0}}
$$

The first three roots correspond to the three branches already found which are finite when $\zeta \rightarrow 0$. The terms in $i \zeta$ reveal that

$$
B=\frac{1}{\alpha_{0}}+\tilde{q}_{1}+\tilde{q}_{2}+\tilde{q}_{3}-\bar{q}_{2}-\bar{q}_{3},
$$

which is positive from (3.9). In terms of $\omega$ the fourth high-frequency branch is therefore

$$
w_{4}(\omega)=-i \omega \frac{\varepsilon \alpha_{1} \tau}{\alpha_{0}}+\frac{1}{\alpha_{0}}+\tilde{q}_{1}+\tilde{q}_{2}+\tilde{q}_{3}-\bar{q}_{2}-\bar{q}_{3}+O\left(\omega^{-1}\right)
$$

$\operatorname{Im} w_{4}(\omega)<0$ and so $w_{4}$ is stable. 
16 of 26

A. M. ALHARBI AND N. H. SCOTT

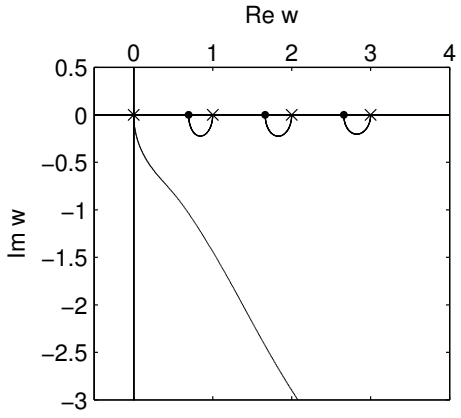

(a) $\tau=0$

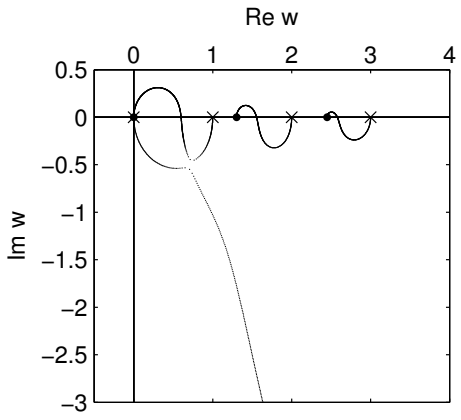

(c) $\tau=0.4$

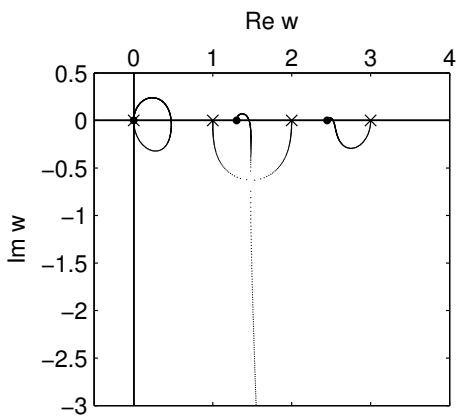

(e) $\tau=1.12$

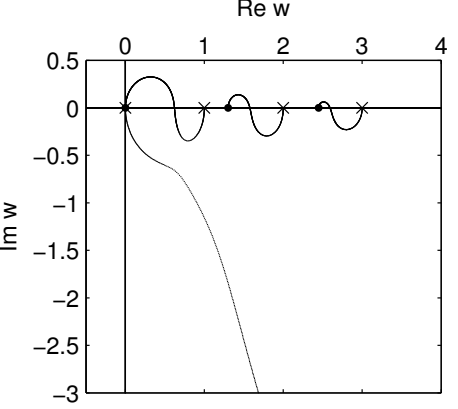

(b) $\tau=0.3$

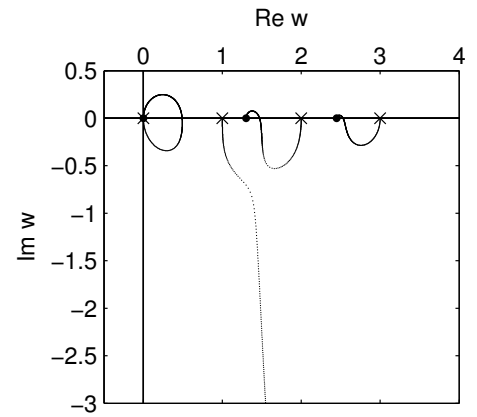

(d) $\tau=1$

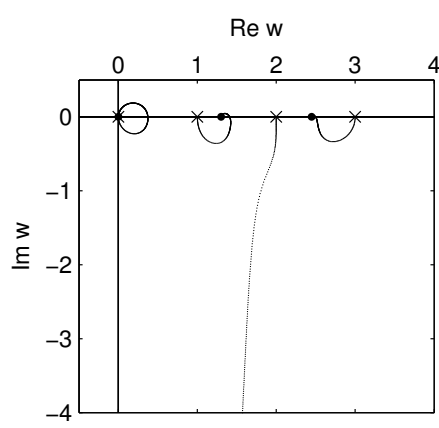

(f) $\tau=2$

FIG. 4. The four błanches of the secular equation for anisotropic generalized temperature-rate-dependent thermoelasticity: Model 1. The case of $\tau$ varying with $\alpha_{0}=0.1, \alpha_{1}=0.2$ fixed. For each part, $\tilde{q}_{1}=0.75, \tilde{q}_{2}=1.75, \tilde{q}_{3}=2.75, \hat{q}_{1}=1, \hat{q}_{2}=2, \hat{q}_{3}=3$. In part (a) $\bar{q}_{1}=0.6952, \bar{q}_{2}=1.6644, \bar{q}_{3}=2.6599, \bar{q}_{4}=11.7307$, but in parts (b)-(f), $\bar{q}_{1}=0, \bar{q}_{2}=1.3021, \bar{q}_{3}=2.4479$. 


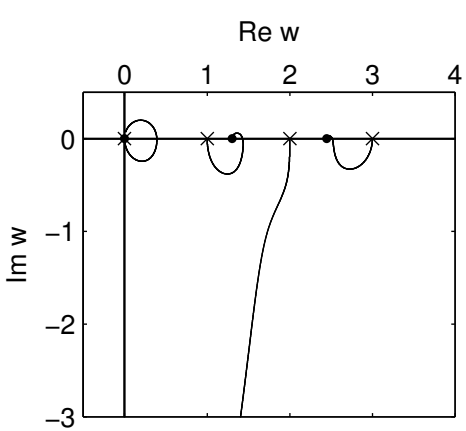

(a) $\tau=0.1$

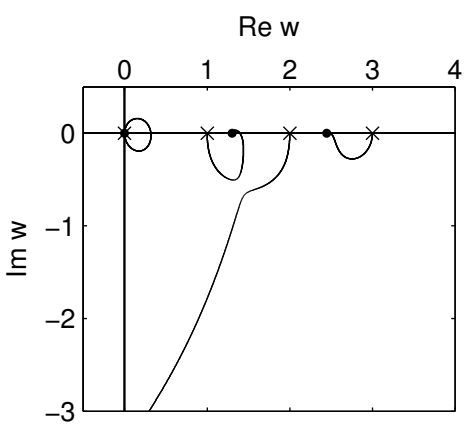

(c) $\tau=1$

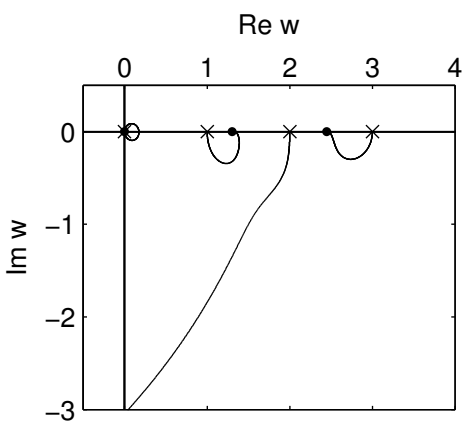

(e) $\tau=5$

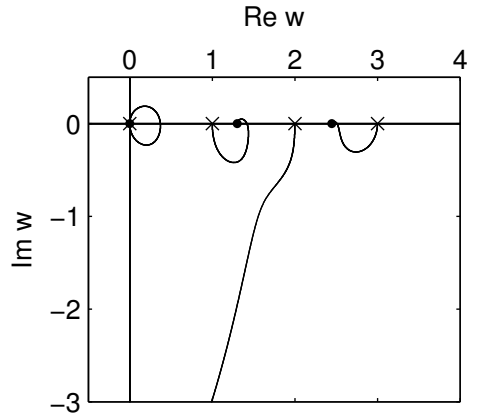

(b) $\tau=0.3$

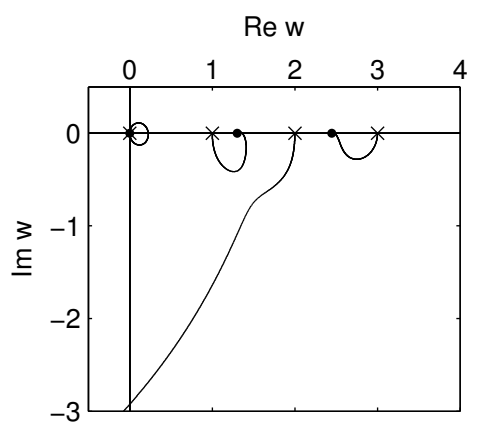

(d) $\tau=3$

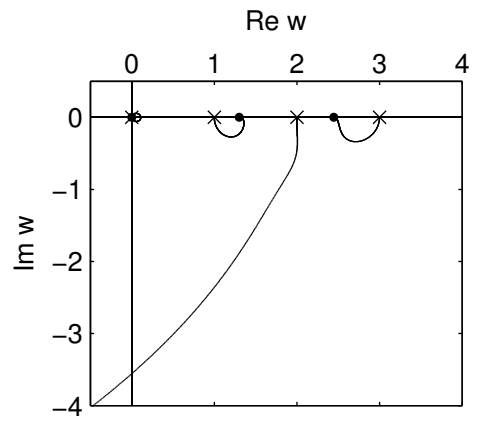

(f) $\tau=10$

FIG. 5. The four branches of the secular equation for anisotropic generalized temperature-rate-dependent thermoelasticity: Model 1 . The case of $\tau$ varying with $\alpha_{0}=0, \alpha_{1}=2$ fixed. For each part, $\tilde{q}_{1}=0.75, \tilde{q}_{2}=1.75, \tilde{q}_{3}=2.75, \hat{q}_{1}=1, \hat{q}_{2}=2, \hat{q}_{3}=3$. 
18 of 26

A. M. ALHARBI AND N. H. SCOTT

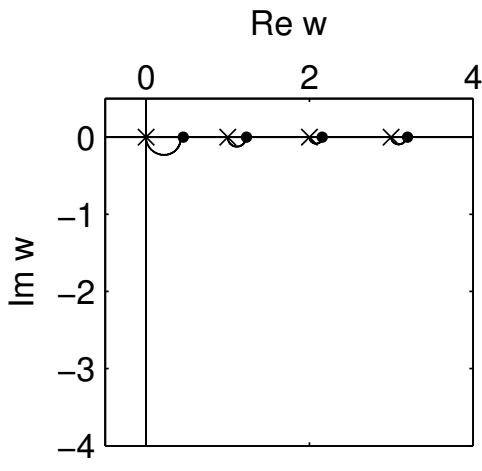

(a) $\tau=0$
Re w

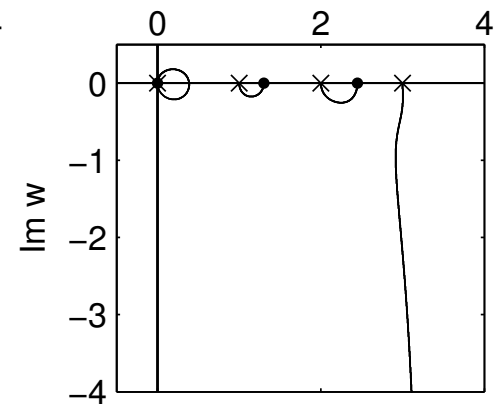

(b) $\tau=0.3$

Re w

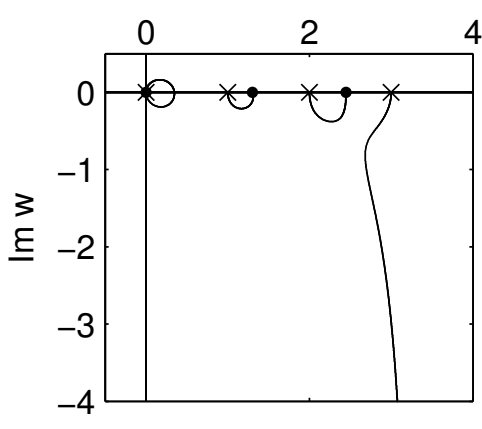

(c) $\tau=0.7$

Re w

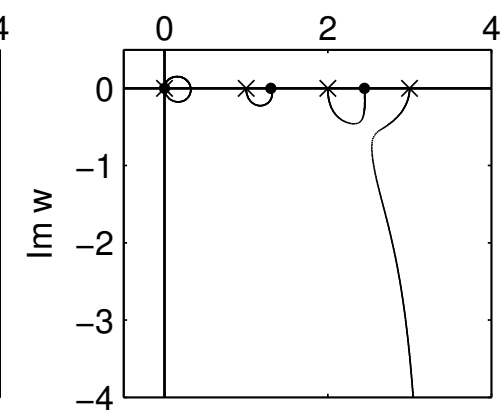

(d) $\tau=1$

Re w

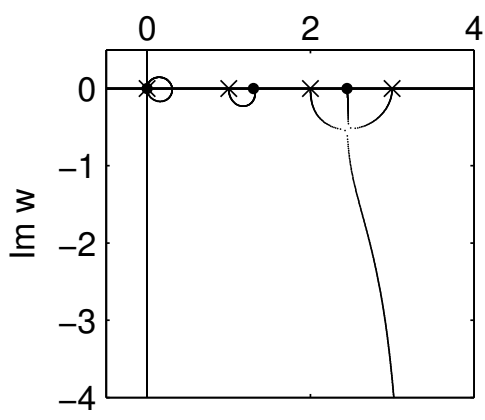

(e) $\tau=1.2$

Re w

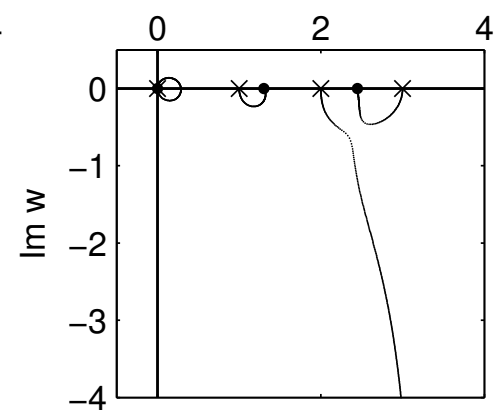

(f) $\tau=1.5$

FIG. 6. The four branches of the secular equation for anisotropic generalized temperature-rate-dependent thermoelasticity: Model 1 . The case of $\tau$ varying with $\alpha_{0}=\alpha_{1}=1$ fixed. For each part, $\tilde{q}_{1}=0.75, \tilde{q}_{2}=1.75, \tilde{q}_{3}=2.75, \hat{q}_{1}=1, \hat{q}_{2}=2, \hat{q}_{3}=3$. 


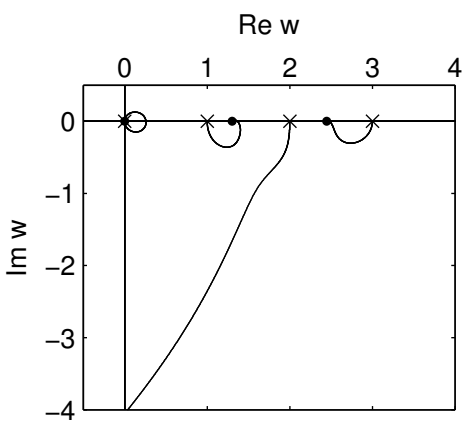

(a) $\alpha_{0}=0.1$

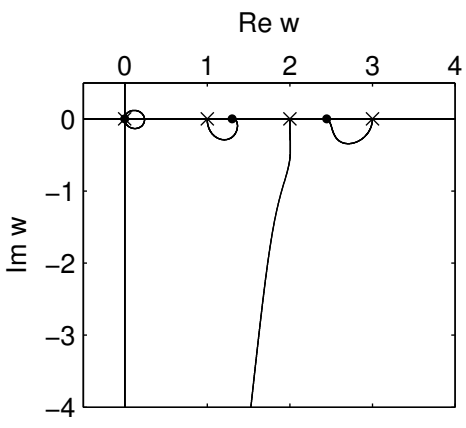

(c) $\alpha_{0}=0.5$

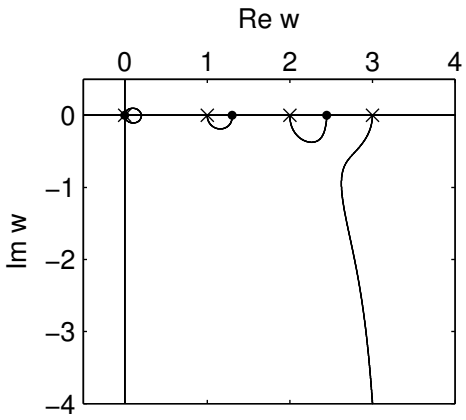

(e) $\alpha_{0}=1.5$

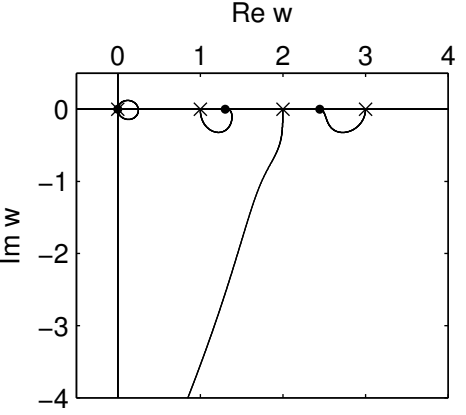

(b) $\alpha_{0}=0.3$

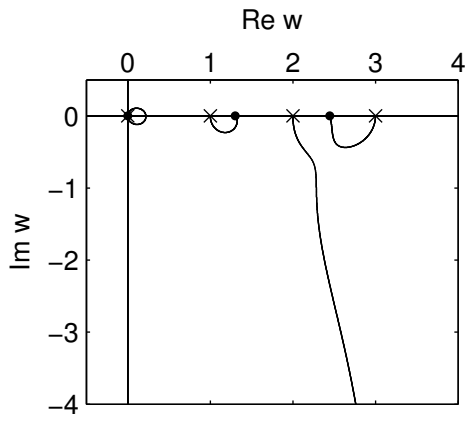

(d) $\alpha_{0}=1$

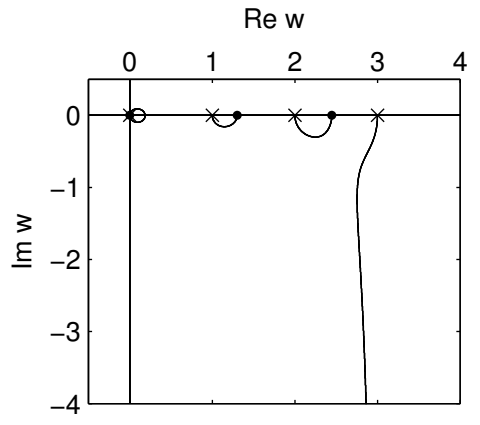

(f) $\alpha_{0}=2$

FIG. 7. The four branches of the secular equation for anisotropic generalized temperature-rate-dependent thermoelasticity: Model 1 . The case of $\alpha_{0}$ varying with $\tau=1, \alpha_{1}=3$ fixed. For each part, $\tilde{q}_{1}=0.75, \tilde{q}_{2}=1.75, \tilde{q}_{3}=2.75, \hat{q}_{1}=1, \hat{q}_{2}=2, \hat{q}_{3}=3$.

We have shown that in the high-frequency limit for Model 1, $w_{1}$ is necessarily unstable, $w_{4}$ is necessarily stable and that the stability or otherwise of $w_{2}$ and $w_{3}$ depends entirely on the magnitude of the relaxation time $\alpha_{0}$ according to (3.17).

For Model 1, we note that branches can change their stability nature for intermediate frequencies, as 
is illustrated in Figures 4-7. This is because we cannot rearrange equation (3.4) to have a real function of $w$ on one side and a real function of $i \omega$ on the other, as we could at (2.37).

\subsection{Numerical results}

In each of Figures 4-7 we used the same values of $\hat{q}_{i}$ and $\tilde{q}_{i}, i=1,2,3$, and various choices for $\tau$, except that in Figure 7, $\tau$ is kept constant. In Figure 4, where $\alpha_{1}>\alpha_{0}>0$, and in Figure 5, where $\alpha_{1}>\alpha_{0}=0$, we have three unstable waves and one stable wave. In Figure 6, where $\alpha_{1}=\alpha_{0}>0$, we have three stable waves and one unstable wave. In Figure 7 we fix $\tau$ and $\alpha_{1}$ and make different choices for $\alpha_{0}$ and find that: in sub-figures (a) and (b), with $\alpha_{0}=0.1$ and 0.3 , respectively, there are three unstable waves and one stable wave; in sub-figure (c), with $\alpha_{0}=0.5$, there are two stable waves and two unstable waves; in sub-figures (d)-(f), with $\alpha_{0}=1,1.5,2$, sequentially, there are three stable waves and one unstable wave. Therefore, the value of $\alpha_{0}$ affects wave stability as predicted by (3.17).

\section{Generalized temperature-rate-dependent thermoelasticity: Model 2}

In the isotropic case, Ignaczak (1980) combined the field equations of temperature-rate-dependent thermoelasticity (2.4) with the modified heat conduction equation (1.2) in order to arrive at a theory of thermoelasticity which we believe to be more rationally based than that discussed in the previous section. Here, we extend Ignaczak's theory to the anisotropic case and refer to it as generalized temperature-ratedependent thermoelasticity: Model 2.

The field equations of the isotropic theory of Ignaczak (1980), when extended to the anisotropic case, become

$$
\begin{aligned}
& \sigma_{i j}=\tilde{c}_{i j k l} u_{k, l}-\beta_{i j}\left(1+\alpha_{1} \frac{\partial}{\partial t}\right) \theta, \\
& \phi=\rho^{-1} \beta_{i j} u_{i, j}+T^{-1} c\left(1+\alpha_{0} \frac{\partial}{\partial t}\right) \theta, \\
& \left(1+\tau \frac{\partial}{\partial t}\right) q_{i}=-k_{i j} \theta_{, j} .
\end{aligned}
$$

Equations $(4.1)_{1,2}$ are equations $(2.2)_{1,2}$ of temperature-rate-dependent thermoelasticity (with $d_{i} \equiv 0$ ) and equation $(4.1)_{3}$ is the heat flux equation (1.2) of generalized thermoelasticity.

In order to derive the field equations of Model 2 we must insert (4.1) into the balance laws (2.1), just as we inserted (2.2) into (2.1) when deriving the field equations of temperature-rate-dependent thermoelasticity. Firstly, by inserting (4.1) $)_{1}$ into the balance of linear momentum $(2.1)_{1}$ we obtain

$$
\rho \ddot{u}_{i}=\tilde{c}_{i j k l} u_{k, l j}-\beta_{i j}\left(1+\alpha_{1} \frac{\partial}{\partial t}\right) \theta_{, j}
$$

which is the same as the corresponding equations $(2.4)_{1}$ and (3.1) $)_{1}$ of the previous two sections. We next eliminate the entropy $\phi$ between $(4.1)_{2}$ and the balance of energy $(2.1)_{2}$ to find that

$$
-q_{i, i}=T \beta_{i j} \dot{u}_{i, j}+\rho c\left(1+\alpha_{0} \frac{\partial}{\partial t}\right) \dot{\theta} .
$$

On differentiating (4.1) $)_{3}$ with respect to $x_{i}$ and using (4.3) to eliminate $q_{i, i}$ we get the fourth field equation in the form

$$
k_{i j} \theta_{, i j}=T \beta_{i j}\left(\dot{u}_{i, j}+\tau \ddot{u}_{i, j}\right)+\rho c\left\{\dot{\theta}+\left(\alpha_{0}+\tau\right) \ddot{\theta}+\alpha_{0} \tau \dddot{\theta}\right\} .
$$


This field equation differs from both $(2.4)_{2}$ and $(3.1)_{2}$, the corresponding field equations of the previous two sections. Equations (4.2) and (4.4) represent the field equations of generalized temperature-ratedependent thermoelasticity: Model 2.

\subsection{The secular equation}

We use the plane harmonic wave forms (2.7) in the field equations (4.2) and (4.4) to reduce them to a linear algebraic system of equations for the amplitudes $U_{i}$ and $\Theta$. Then, arguing as in the previous two sections, and using the dimensionless quantities (2.13) and (3.3), we obtain the dimensionless secular equation

$$
\hat{F}(w)+i \omega \frac{1+w\left(\alpha_{1}-\alpha_{0}\right)(1-i \omega \tau)}{\left(1-i \omega \alpha_{1}\right)(1-i \omega \tau)} \tilde{G}(w)=0,
$$

in which $\hat{F}(w)$ and $\tilde{G}(w)$ are defined at (2.22). This is the dimensionless secular equation of generalized temperature-rate-dependent thermoelasticity: Model 2. On putting $\tau=0$ in (4.5) we get the corresponding secular equation (2.21) of temperature-rate-dependent thermoelasticity, as expected, and on putting $\alpha_{0}=\alpha_{1}=0$, we get the secular equation of generalized thermoelasticity, see (Scott, 1989b, Equation (4)), again as expected.

\subsubsection{The uncoupled case $\varepsilon=0 . \quad$ In this case the roots of the secular equation (4.5) become}

$$
w_{0}=\frac{-i \omega}{\left(1-i \omega \alpha_{0}\right)\left(1-i \omega \tau_{1}\right)}, \quad w_{i}=\tilde{q}_{i}=\hat{q}_{i}, \quad i=1,2,3,
$$

the last three corresponding to unattenuated, non-dispersive purely elastic modes as at equation (2.25). On decomposing (4.6) 1 into its real and imaginary parts $w_{0}=u_{0}+i v_{0}$ we find on eliminating $\omega$ that $w_{0}$ describes the full circle

$$
\left(u_{0}-\frac{1}{2\left(\alpha_{0}+\tau_{1}\right)}\right)^{2}+v_{0}^{2}=\left(\frac{1}{2\left(\alpha_{0}+\tau_{1}\right)}\right)^{2}
$$

in the complex $w$-plane as $\omega$ ranges over positive values, beginning at the origin $(\omega=0)$ and finishing also at the origin $(\omega \rightarrow \infty)$. The real axis is intersected at $u_{0}=1 /\left(\alpha_{0}+\tau_{1}\right), v_{0}=0$, corresponding to the frequency $\omega=\omega_{c} \equiv 1 / \sqrt{\alpha_{0} \tau_{1}}$. The branch $w_{0}$ is stable for $\omega<\omega_{c}$ but is unstable for $\omega>\omega_{c}$.

\subsection{Low-frequency expansions}

When $\omega=0$, the roots of the secular equation (4.5) are the zeros of $\hat{F}(w)$, namely, $w=\hat{q}_{i}, i=0,1,2,3$, defining $\hat{q}_{0} \equiv 0$, exactly as in the previous two sections. Taylor expansions of the roots of equation (4.5) for small $\omega$ reveal that

$$
w_{i}(\omega)=\hat{q}_{i}-i \omega\left\{1+\hat{q}_{i}\left(\alpha_{1}-\alpha_{0}\right)\right\} \frac{\tilde{G}\left(\hat{q}_{i}\right)}{\hat{F}^{\prime}\left(\hat{q}_{i}\right)}+O\left(\omega^{2}\right), \quad i=0,1,2,3,
$$

which is exactly the same as (2.26) in the temperature-rate-dependent case. Thus the relaxation time $\tau$ does not appear at $O(\omega)$ in this theory.

When $i=0$, so that $\hat{q}_{0}=0,(4.8)$ reduces to

$$
w_{0}(\omega)=-i \omega \frac{\tilde{G}(0)}{\hat{F}^{\prime}(0)}=-i \omega \frac{\tilde{q}_{1} \tilde{q}_{2} \tilde{q}_{3}}{\hat{q}_{1} \hat{q}_{2} \hat{q}_{3}},
$$


ignoring $O\left(\omega^{2}\right)$ terms. It is clear that $\operatorname{Im} w_{0}<0$ and so $w_{0}$ is stable.

For $i=1,2,3,(2.28)$ holds as before and so there are once again four stable waves in the lowfrequency limit.

\subsection{High-frequency expansions}

The roots of the secular equation (4.5) in the high-frequency limit $\omega \rightarrow \infty$ are best examined by writing $\omega=1 / \zeta$ and taking the limit $\zeta \rightarrow 0$, as before. The secular equation (4.5) then becomes

$$
\hat{F}(w)-\frac{w \tau\left(\alpha_{1}-\alpha_{0}\right)+i \zeta\left\{1+w\left(\alpha_{1}-\alpha_{0}\right)\right\}}{\alpha_{1} \tau\left(1+i \zeta / \alpha_{1}\right)(1+i \zeta / \tau)} \tilde{G}(w)=0,
$$

valid for all $\zeta$.

At $\zeta=0,(4.10)$ reduces to

$$
H(w) \equiv \hat{F}(w)-\left(1-\alpha_{0} / \alpha_{1}\right) w \tilde{G}(w)=0 .
$$

This equation is quartic in $w$ with roots denoted by $\bar{q}_{0}, \bar{q}_{1}, \bar{q}_{2}, \bar{q}_{3}$, with $\bar{q}_{0} \equiv 0$. By examining the sign changes of $H(w)$ we discover the further interlacing properties

$$
0=\bar{q}_{0}<\tilde{q}_{1}<\hat{q}_{1}<\bar{q}_{1}<\tilde{q}_{2}<\hat{q}_{2}<\bar{q}_{2}<\tilde{q}_{3}<\hat{q}_{3}<\bar{q}_{3} .
$$

The real quartic polynomial

$$
\bar{h}(w)=w\left(w-\bar{q}_{1}\right)\left(w-\bar{q}_{2}\right)\left(w-\bar{q}_{3}\right)
$$

must be a scalar multiple of $H(w)$ because both have the same four zeros:

$$
H(w)=\frac{\alpha_{0}}{\alpha_{1}} \bar{h}(w)
$$

But from (4.11) and (4.13), equation (4.10) reduces to

$$
\bar{h}(w)-\frac{i \zeta}{\alpha_{0} \tau}\left\{1-w \tau\left(1-\alpha_{0} / \alpha_{1}\right)\right\} \tilde{G}(w)+O\left(\zeta^{2}\right)=0,
$$

because only $O(i \zeta)$ terms need be retained. Taylor expansions of (4.14) show that

$$
w_{i}(\omega)=\bar{q}_{i}+\frac{i \omega^{-1}}{\alpha_{0} \tau}\left\{1-\bar{q}_{i} \tau\left(1-\alpha_{0} / \alpha_{1}\right)\right\} \frac{\tilde{G}\left(\bar{q}_{i}\right)}{\bar{h}^{\prime}\left(\bar{q}_{i}\right)}+O\left(\omega^{-2}\right), \quad i=0,1,2,3,
$$

reverting to the notation $\zeta=\omega^{-1}$. The interlacing inequalities (4.12) show that

$$
\frac{\tilde{G}\left(\bar{q}_{i}\right)}{\bar{h}^{\prime}\left(\bar{q}_{i}\right)}>0, \quad i=0,1,2,3 .
$$

When $i=0$, so that $\bar{q}_{0}=0,(4.15)$ reduces to

$$
w_{0}(\omega)=\frac{i \omega^{-1}}{\alpha_{0} \tau} \frac{\tilde{G}(0)}{\bar{h}^{\prime}(0)}=\frac{i \omega^{-1}}{\alpha_{0} \tau} \frac{\tilde{q}_{1} \tilde{q}_{2} \tilde{q}_{3}}{\bar{q}_{1} \bar{q}_{2} \bar{q}_{3}}
$$

ignoring $O\left(\omega^{-2}\right)$ terms. We see that $\operatorname{Im} w_{0}>0$ and so $w_{0}$ is unstable. 


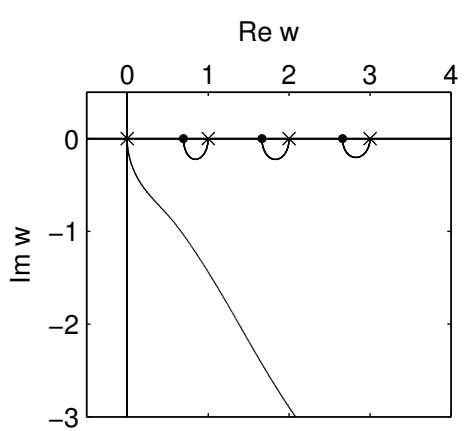

(a) $\tau=0$

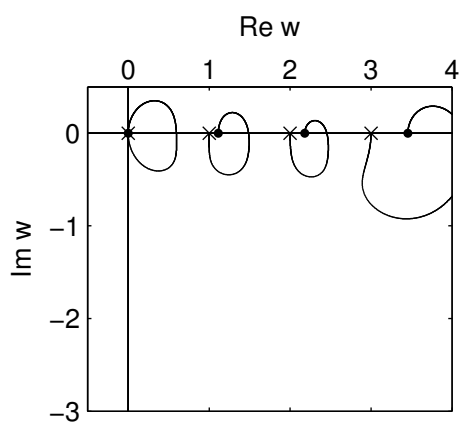

(c) $\tau=0.3$

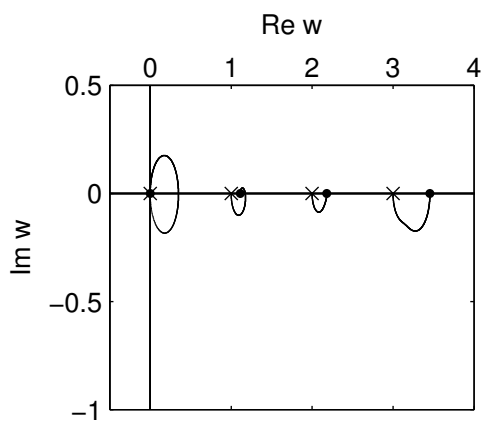

(e) $\tau=1.2$

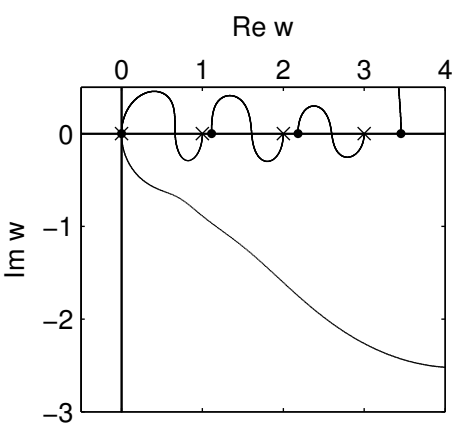

(b) $\tau=0.1$

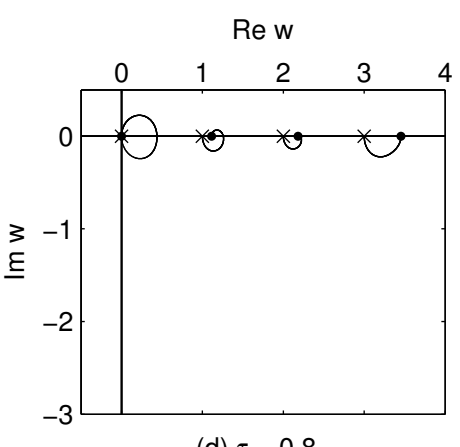

(d) $\tau=0.8$

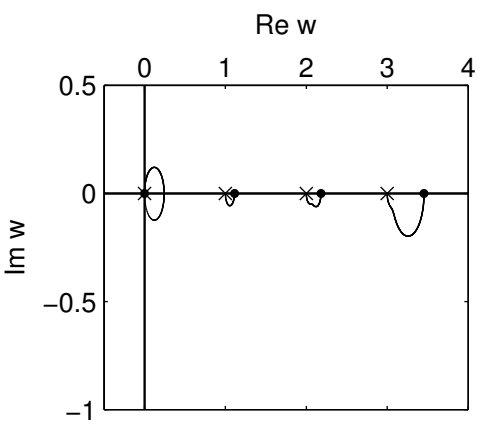

(f) $\tau=2$

FIG. 8. The four branches of the secular equation for anisotropic generalized temperature-rate-dependent thermoelasticity: Model 2. The case of $\tau$ varying with $\alpha_{0}=0.1, \alpha_{1}=0.2$ fixed. For each part, $\tilde{q}_{1}=0.75, \tilde{q}_{2}=1.75, \tilde{q}_{3}=2.75, \hat{q}_{1}=1, \hat{q}_{2}=2, \hat{q}_{3}=3$. In part (a) $\bar{q}_{1}=0.6952, \bar{q}_{2}=1.6644, \bar{q}_{3}=2.6599, \bar{q}_{4}=11.7307$, but in parts (b)-(f), $\bar{q}_{1}=0, \bar{q}_{2}=1.1131, \bar{q}_{3}=2.1816, \bar{q}_{4}=3.4553$.

For the other three branches of (4.15) we conclude from (4.16) that $w_{i}$ is stable if

$$
1-\bar{q}_{i} \tau\left(1-\alpha_{0} / \alpha_{1}\right)<0, \quad i=1,2,3
$$


Let us define the relaxation times

$$
\tau_{i} \equiv \frac{1}{\bar{q}_{i}\left(1-\alpha_{0} / \alpha_{1}\right)}, \quad i=1,2,3 .
$$

Since $\bar{q}_{1}<\bar{q}_{2}<\bar{q}_{3}$ we can say that $\tau_{1}>\tau_{2}>\tau_{3}$. As $\tau$ varies we see that for

$$
\begin{array}{ll}
\tau>\tau_{1}>\tau_{2}>\tau_{3}, & \text { all three branches are stable, } \\
\tau_{1}>\tau>\tau_{2}>\tau_{3}, & w_{1} \text { is unstable but } w_{2}, w_{3} \text { are stable, } \\
\tau_{1}>\tau_{2}>\tau>\tau_{3}, & w_{1}, w_{2}, \text { are unstable but } w_{3} \text { is stable, } \\
\tau_{1}>\tau_{2}>\tau_{3}>\tau, & \text { all three branches are unstable. }
\end{array}
$$

Thus, all branches are stable when $\tau$ is large enough but all are unstable if $\tau$ is small enough. However, if $\tau=0$ the situation reverts to the temperature-rate-dependent thermoelasticity of Section 2 and all branches are stable.

\subsection{Numerical results}

In each part of Figure 8 , the eigenvalues $\hat{q}_{i}$ and $\tilde{q}_{i}, i=1,2,3$, are the same as are the relaxation times $\alpha_{0}$ and $\alpha_{1}$. Low-frequency limits are marked with a $\times$ and high-frequency limits with a $\bullet$. The relaxation time $\tau$ increases from sub-figure to sub-figure. In Fig. 8(a), where $\tau=0$, there are four stable waves as this is the case of temperature-rate-dependent thermoelasticity. As $\tau$ increases slightly from zero we see three unstable waves in parts (b) and (c). As $\tau$ increases further we see more stable modes until there is only one unstable mode in Fig. 8(f). This is in accord with the predictions of inequalities (4.18).

\section{Discussion}

We saw in Section 2 that the Green \& Lindsay (1972) theory of temperature-rate-dependent thermoelasticity is stable in the fully anisotropic case, that is, the amplitude of an initially small disturbance remains uniformly bounded in the direction of travel for all time. Leslie \& Scott (1998) have previously demonstrated the stability of classical thermoelasticity in the isotropic case, see also Chadwick (1973), and Scott (1989a) in the anisotropic case, so that the present work is an extension of these results. Furthermore, Scott (1989b) and Leslie \& Scott (2000) have demonstrated the stability of generalized thermoelasticity in which Fourier's law of heat conduction (1.1) is replaced by (1.2), thereby introducing a relaxation time. The present work is also an extension of these results.

In any material we might expect an initial small disturbance either to decay to zero or remain bounded as time increases. Thus we might regard the stability of wave forms as being a possible criterion for physically reasonable response. We have seen that both classical and generalized thermoelasticity possess this property and we showed in section 2 that the Green \& Lindsay (1972) theory of temperature-rate-dependent thermoelasticity also shares this stability property and so might be an acceptable non-classical model for thermoelsaticity on grounds of physically reasonable response .

In Section 3, however, we saw that the model we proposed for generalized temperature-rate-dependent thermoelasticity on the basis of the Chandrasekharaiah \& Keshavan (1991) unified system of governing equations is not stable, one of the four waves being necessarily stable, another being necessarily unstable and the other two possibly being unstable at high frequencies but both stable at low frequencies. Therefore, generalized temperature-rate-dependent thermoelasticity: Model 1 does not possess the stability property and so we might conclude that this is not a good physical model on grounds of lack of 
physically reasonable response; this is hardly surprising as it is based on a system of equations, namely (3.1), that has no physical basis.

In Section 4 we considered an alternative theory of generalized temperature-rate-dependent thermoelasticity put forward originally by Ignaczak (1980) for the isotropic case, in which he combined the field equations of temperature-rate-dependent thermoelasticity (2.2) with the heat conduction equation (1.2) of generalized thermoelasticity. We saw that this procedure too led to one of the four waves being unstable and the other three being stable or unstable depending on the value of the relaxation time $\tau$. This lack of stability is somewhat surprising as Ignacak's theory is rationally based in that our field equations (4.1) were obtained in exactly the same way that the (stable wave) theory of generalized thermoelasticity, see Scott (1989b), was obtained from the classical thermoelasticity equations (2.5) and the heat conduction equation of generalized thermoelasticity (1.2).

\section{Acknowledgement}

A.M. Alharbi wishes to thank Taif University and the Saudi Arabian Ministry of Higher Education for their financial support during her PhD studies.

\section{REFERENCES}

CHAdwick, P. (1973) Interchange of modal properties in the propagation of harmonic waves in heat-conducting materials. Bull. Austral. Math. Soc., 8, 75-92.

CHADWICK, P. (1979) Basic properties of plane harmonic waves in a prestressed heat-conducting elastic material. J.Thermal Stresses, 2, 193-214.

Chandrasekharaiah, D. S. (1986) Thermoelasticity with second sound: A review. Applied Mechanics Reviews, 39, 355-376.

Chandrasekharaiah, D. S. \& Keshavan, H. R. (1991) Thermoelastic plane waves in a transversely isotropic body. Acta Mechanica, 87, 11-22.

Green, A. E. \& Linds AY, K. A. (1972) Thermoelasticity. J. Elasticity, 2, 1-7.

IGNACZAK, J. (1980) Linear dynamic thermoelasticity-a survey. Shock Vibration Digest, 13, 3-8.

LESLIE, D. J. \& SCOTT, N. H. (1998) Incompressibility at uniform temperature or entropy in isotropic thermoelasticity, Q. JI Mech. Appl. Math., 51, 191-211.

Leslie, D. J. \& ScotT, N. H. (2000) Wave stability for incompressibility at uniform temperature or entropy in generalized isotropic thermoelasticity, Q. JI Mech. Appl. Math., 53, 1-25.

Lord, H. W. \& Shulman, Y. (1967) A generalized dynamical theory of thermoelasticity, J. Mech. Phys. Solids, 15, 299-309.

Straughan, B. (2011) Heat Waves. New York, Dordrecht, Heidelberg, London: Springer.

Scott, N. H. (1989a) A theorem in thermoelasticity and its application to linear stability. Proc. R. Soc. Lond. A, 424, 143-153.

Scotт, N. H. (1989b) The stability of plane waves in generalised thermoelasticity. In Elastic Wave Propagation, Eds M. F. McCarthy and M. A. Hayes, pp 623-628. North-Holland, Amsterdam.

\section{A. Derivation of the secular equation (2.12)}

Written in matrix form equations (2.11) become

$$
\left[\begin{array}{cccc}
\tilde{Q}_{11}-\rho s^{-2} & \tilde{Q}_{12} & \tilde{Q}_{13} & i \omega^{-1} s^{-1} b_{1}\left(1-i \omega \alpha_{1}\right) \\
\tilde{Q}_{21} & \tilde{Q}_{22}-\rho s^{-2} & \tilde{Q}_{23} & i \omega^{-1} s^{-1} b_{2}\left(1-i \omega \alpha_{1}\right) \\
\tilde{Q}_{31} & \tilde{Q}_{32} & \tilde{Q}_{33}-\rho s^{-2} & i \omega^{-1} s^{-1} b_{3}\left(1-i \omega \alpha_{1}\right) \\
T s^{-1} b_{1} & T s^{-1} b_{2} & T s^{-1} b_{3} & k-i \omega^{-1}\left(1-i \omega \alpha_{0}\right) c \rho s^{-2}
\end{array}\right]\left[\begin{array}{c}
U_{1} \\
U_{2} \\
U_{3} \\
\Theta
\end{array}\right]=0 .
$$


These equations have non-zero solutions for the amplitudes $U_{1}, U_{2}, U_{3}, \Theta$ if and only if the determinant of the above $4 \times 4$ matrix vanishes. This determinant condition may be written in the form

$$
\left|\begin{array}{cc}
\tilde{\mathbf{Q}}-w \mathbf{1} & \bar{\alpha} \mathbf{b} \\
\beta \mathbf{b}^{T} & \gamma_{1}
\end{array}\right|=0
$$

where we have defined

$$
w=\rho s^{-2}, \bar{\alpha}=i \omega^{-1} s^{-1}\left(1-i \alpha_{1} \omega\right), \beta=T s^{-1}, \gamma_{1}=k-i \omega^{-1}\left(1-i \omega \alpha_{0}\right) c w .
$$

We can rewrite (A.1) as

$$
D \equiv\left|\begin{array}{cc}
\tilde{\mathbf{Q}}-w \mathbf{1} & \bar{\alpha} \mathbf{b}+\mathbf{0} \\
\beta \mathbf{b}^{T} & -\delta+\left(\gamma_{1}+\delta\right)
\end{array}\right|=0,
$$

in which $\delta$ is, so far, an arbitrary quantity. Using properties of determinants to expand by the fourth column we have

$$
D=\left|\begin{array}{cc}
\tilde{\mathbf{Q}}-w \mathbf{1} & \bar{\alpha} \mathbf{b} \\
\beta \mathbf{b}^{T} & -\delta
\end{array}\right|+\left|\begin{array}{cc}
\tilde{\mathbf{Q}}-w \mathbf{1} & \mathbf{0} \\
\beta \mathbf{b}^{T} & \gamma_{1}+\delta
\end{array}\right| .
$$

The first determinant, denoted by $D_{1}$, may be simplified by adding to row $i$, for $i=1,2,3$, the multiple $\bar{\alpha} b_{i} / \delta$ of row 4 , to get

$$
D_{1}=\left|\begin{array}{cc}
\tilde{\mathbf{Q}}-w \mathbf{1}+\frac{\bar{\alpha} \beta}{\delta} \mathbf{b} \otimes \mathbf{b} & \mathbf{0} \\
\beta \mathbf{b}^{T} & -\delta
\end{array}\right|=-\delta \operatorname{det}\left(\tilde{\mathbf{Q}}-w \mathbf{1}+\frac{\bar{\alpha} \beta}{\delta} \mathbf{b} \otimes \mathbf{b}\right),
$$

expanding the determinant by the fourth column. In order to force $D_{1}$ to be defined in terms of $\hat{\mathbf{Q}}$ in equation (A.4) we compare with equation $(2.10)_{2}$ to obtain

$$
\frac{T}{\rho c}=\frac{\bar{\alpha} \beta}{\delta},
$$

which from (A.2) 2,3 fixes the value of $\delta$ to be

$$
\delta=i \omega^{-1}\left(1-i \omega \alpha_{1}\right) c w
$$

The second determinant of (A.3), denoted by $D_{2}$, is

$$
D_{2}=\left(\gamma_{1}+\delta\right) \operatorname{det}\{\tilde{\mathbf{Q}}-w \mathbf{1}\}
$$

again expanding by the fourth column. On dividing the determinant $D=D_{1}+D_{2}$ by $\left(-i \omega^{-1}(1-\right.$ $\left.i \omega \alpha_{1}\right) c$ ) we find that, after further reduction, equation (A.1) is equivalent to the secular equation (2.12). 